\title{
Potential Measures for Spectrally Negative Markov Additive Processes with Applications in Ruin Theory
}

\author{
Runhuan Feng* and Yasutaka Shimizu ${ }^{\dagger}$
}

July 28,2014

\begin{abstract}
The Markov additive process (MAP) has become an increasingly popular modeling tool in the applied probability literature. In many applications, quantities of interest are represented as functionals of MAPs and the potential measure, also known as the resolvent measure, have played a key role in the representations of explicit solutions to these functionals. In this paper, closed-form solutions to potential measures for spectrally negative MAPs are found using a novel approach based on algebraic operations of matrix operators. This approach also provides a connection between results from fluctuation theoretic techniques and those from classical differential equation techniques. In the end, the paper presents a number of applications to ruin-related quantities as well as verification of known results concerning exit problems.
\end{abstract}

Keywords: Markov additive processes, potential measure, resolvent density, Markov renewal equation, scale matrix, exit problems.

MSC2010: 60J25, 91B30, 60J28.

\section{Introduction}

In the recent applied probability literature, there are two notably different lines of development for exit problems of Lévy processes and Markov additive processes, with a considerable amount of applications in ruin theory.

Historically, classical first passage time problems are often solved by differential equations and integro-differential equations. Many authors have developed solution methods for various risk models with an increasing use of differential and integral operators. See, for example, Li and Garrido [37], Gerber and Shiu [24], Landriault and Willmot [36], Cheung and Landriault [15], Cai et al. [11], Zhang and Cheung [39], etc. The recent work by Albrecher et al. [2] and Albrecher et al. [1] sheds light on the connection of these applications with the study of integro-differential algebras. See details in Rosenkranz and Regensburger [38].

There is also a growing popularity in applications of fluctuation theoretical techniques to ruin-related problems, which employ entirely different solution methods such as martingales and changes of measures. See for example, Avram, Palmowski and Pistorius [5], Breuer [9, 10], Kyprianou and Zhou [35], etc. Interested readers may consult Kyprianou [32] and Asmussen and Albrecher [4] for comprehensive accounts of these techniques and applications in ruin theory.

Despite the overlap of their research aims, there are relatively few papers that make connections between the two approaches. Some earlier attempts to connect the two approaches can be

\footnotetext{
${ }^{*}$ Department of Mathematics, University of Illinois at Urbana-Champaign, 1409 W. Green Street, M/C 382, Urbana, IL 61801, USA; rfeng@illinois.edu

${ }^{\dagger}$ Department of Applied Mathematics, Waseda University 3-4-1, Okubo, Shinjuku, Tokyo 169-8555, Japan. shimizu@waseda.jp
} 
seen in Biffis and Kyprianou [7], Biffis and Morales [8], Feng and Shimizu [22] in various settings for spectrally negative Lévy processes. In this paper, we intend to establish some equivalency of results from fluctuation techniques and those from integro-differential operator techniques in the context of MAP risk models.

The key to analyzing each stochastic process often involves studying its semigroup. It is well known that every transition density function defines a contraction semigroup (c.f. Theorem II.2.1 of [17]). For any Borel measurable function $l$,

$$
\mathcal{P}_{t} l(x)=\mathbb{E}^{x}\left[l\left(X_{t}\right)\right] .
$$

In the applied probability literature, it is often important to find explicit expressions for the resolvent operator (the corresponding measure is called the potential measure), which is known to be the Laplace transform of the semigroup.

$$
\mathcal{R}_{\delta} l(x)=\int_{0}^{\infty} e^{-\delta t} \mathcal{P}_{t} l(x) \mathrm{d} t, \quad \delta>0 .
$$

In the context of ruin theory, different expressions of the resolvent operator are known for the two above-mentioned methods, which elucidates differences between the two theories. We quote an example from spectrally negative Lévy risk models denoted by $X=\left\{X_{t}, t \geq 0\right\}$ with characteristics $\left(\mu, \sigma^{2}, \nu\right)$ killed when exiting $[0, \infty)$.

In the applications of fluctuation theory, solutions are typically represented in terms of the scale function, which is defined via its Laplace transform

$$
\int_{0}^{\infty} e^{-\lambda x} W^{(\delta)}(x) \mathrm{d} x=\frac{1}{\psi(\lambda)-\delta},
$$

where $\psi(\lambda)=\log \mathbb{E}\left[\exp \left(\lambda X_{1}\right)\right]$. It is well-known that (c.f. Kyprianou [32, Corollary 8.8])

$$
\mathcal{R}_{\delta} l(x)=\int_{0}^{\infty} r^{(\delta)}(x, y) l(y) \mathrm{d} y,
$$

where $r^{(\delta)}$ is known as the potential density

$$
r^{(\delta)}(x, y)=e^{-\rho y} W^{(\delta)}(x)-W^{(\delta)}(x-y), \quad x, y \geq 0 .
$$

The constant $\rho$ is the unique non-negative root of the equation $\psi(\rho)-\delta=0$.

In contrast, the exposition of solutions to ruin-related quantities in classic ruin theory often hinges on compound geometric distributions. For brevity, we reiterate a special case in Feng and Shimizu [22] where $\sigma>0$ and $\int_{0}^{1} z \nu(\mathrm{d} z)<\infty$. (The corresponding result for $\sigma=0$ is also provided in the paper.) The associated compound geometric distribution is given by

$$
G_{\delta}(y)=(1-p)+\sum_{k=1}^{\infty}(1-p) p^{k} \int_{0}^{x} q_{\delta}^{* k}(y) \mathrm{d} y,
$$

where $*$ denotes convolution and

$$
p:=\frac{2}{\sigma^{2}} \int_{0}^{\infty} \mathcal{E}_{\beta}\left\{\mathcal{T}_{\rho} \nu\right\}(y) \mathrm{d} y \quad q_{\delta}(y):=\frac{2}{p \sigma^{2}} \mathcal{E}_{\beta}\left\{\mathcal{T}_{\rho} \nu\right\}(y) .
$$

and $\beta:=2 c / \sigma^{2}+\rho$. The two integral operators involved are defined by

$$
\mathcal{E}_{s} f(x):=e^{-s x} \int_{0}^{x} e^{s y} f(y) \mathrm{d} y ; \quad \mathcal{T}_{s} \nu(x):=e^{s x} \int_{x}^{\infty} e^{-s y} \nu(\mathrm{d} y) .
$$


It is shown that the potential density has the series representation

$$
r^{(\delta)}(x, y)=\frac{1}{1-p} \int_{[0, x)} B_{\delta}(x-z, y) \mathrm{d} G_{\delta}(z)
$$

where

$$
B_{\delta}(x, y)=\left\{\begin{array}{lc}
\frac{2}{\sigma^{2}(\beta+\rho)} e^{-\beta x}\left(e^{\beta y}-e^{-\rho y}\right), & 0<y<x \\
\frac{2}{\sigma^{2}(\beta+\rho)}\left(e^{\rho x}-e^{-\beta x}\right) e^{-\rho y}, & x \leq y .
\end{array}\right.
$$

Note, however, Feng and Shimizu [22] did not just stop at this class of Lévy process for which (1.3) holds but also used an operator-based method to prove (1.2) in the general case, which was only known previously via fluctuation methods.

To bridge the gap between the two types of results in (1.2) and (1.3), Feng and Shimizu [22] proved their equivalence through the representation of the scale function in terms of the compound geometric distribution

$$
W^{(\delta)}(x)=\frac{2}{\sigma^{2}(1-p)(\beta+\rho)} \int_{[0, x)}\left(e^{\rho(x-y)}-e^{-\beta(x-y)}\right) \mathrm{d} G_{\delta}(y) .
$$

The primary goals of this paper can be summarized as follows.

1. We aim to identify the potential measure for spectrally negative Markov additive processes (MAPs), of which less is known in the literature compared with Lévy processes. It was brought to our attention recently that the expression of the potential density in Theorem 4.2 was also found in Ivanovs [27] with fluctuation methods. Nevertheless, this work is entirely based on operator calculation and gives more explicit expressions in certain cases. The operator-based approach leads to a natural extension of classical ruin methods.

2. We are also interested in the analogue of (1.4) for the MAP risk model, which appears to be a key quantity in connecting the seemingly different yet equivalent solutions from classical ruin methods and fluctuation methods.

The rest of this paper is organized as follows. We introduce the notation of the MAP risk model in Section 2.1 and present the main results of the paper in Section 2.2, in which major steps of the matrix-operator approach are outlined in Section 3. As a consequence, in Section 4 , we derive the potential densities for the MAP risk model in two cases, which are essentially the analogues of (1.2) and (1.3). In Section 5, we present some new results for ruin-related problems and also verify through a few examples that the results produced from the matrixoperator approach are consistent with those from Kyprianou and Palmowski [34].

\section{Markov additive risk model}

\subsection{Models and notation}

Let $(\Omega, \mathcal{F}, \mathbb{P})$ be a probability space on which the following stochastic processes are defined. A Markov additive process is defined as a bivariate Markov process $\left\{\left(X_{t}, J_{t}\right), t \geq 0\right\}$, where $J=\left\{J_{t}, t \geq 0\right\}$ is a Markov process with a state space $E$ and the increments of $X=\left\{X_{t}, t \geq 0\right\}$ are governed by $\left\{J_{t}\right\}$ in the sense that for all bounded measurable functions $f$ and $g$,

$$
\mathbb{E}\left[f\left(X_{t+s}-X_{t}\right) g\left(J_{t+s}\right) \mid \mathcal{F}_{t}\right]=\mathbb{E}^{\left(0, J_{t}\right)}\left[f\left(X_{s}\right) g\left(J_{s}\right)\right],
$$

with probability measures $\left\{\mathbb{P}^{(x, i)}, x \in \mathbb{R}, i \in E\right\}$ under which $\mathbb{P}^{(x, i)}\left\{X_{0}=x, J_{0}=i\right\}=1$. A subordinator is an almost surely non-decreasing Lévy process. Any non-subordinator Lévy 
process with no negative jumps is known as a spectrally positive Lévy (SPL) process. In this paper, we consider a fairly general class of risk models known as the MAP risk model in ruin theory. Let $J$ be an irreducible continuous-time Markov chain with state space $E=\{1,2, \cdots, m\}$ and infinitesimal generator matrix $\Lambda=\left(\lambda_{i j}\right)_{i, j \in E}$ with $\lambda_{i i}=-\sum_{i \neq j} \lambda_{i j}<0$. For $J_{t}=i$, the level process $X$ is given by a Lévy process with finite mean $X_{t}^{(i)}=\mu_{i} t-S_{t}^{(i)}-Z_{t}^{(i)}$, where $S^{(i)}$ is a subordinator and $Z^{(i)}$ is a mean zero SPL process. In the context of ruin theory, $X$ is often interpreted as the surplus of an insurance business with $\mu_{i}$ denoting the rate at which premiums are collected under the economy state $i,\left\{S_{t}^{(i)}\right\}$ denoting the aggregate claim costs and $\left\{Z_{t}^{(i)}\right\}$ denoting a perturbation caused by unexpected losses. Hence $X$ has the Lévy exponent

$$
\psi_{i}(s)=\mu_{i} s+\frac{1}{2} \sigma_{i}^{2} s^{2}+\int_{0}^{\infty}\left(e^{-s z}-1\right) \nu_{i}^{S}(d z)+\int_{0}^{\infty}\left(e^{-s z}-1+s z\right) \nu_{i}^{Z}(d z),
$$

where $\nu_{i}^{S}$ and $\nu_{i}^{Z}$ are the corresponding Lévy measures for $S^{(i)}$ and $Z^{(i)}$ respectively, and $\sigma_{i} \geq 0$ is the volatility coefficient for the Gaussian component of $Z^{(i)}$. Note that the finite mean of $X$ implies that $\int_{1}^{\infty} z \nu_{i}^{Z}(d z)<\infty$, which explains why the usual indicator $I(z<1)$ in the Lévy exponent is removed. Throughout the paper, we assume

$$
-X^{(i)}(i=1, \ldots, m) \text { is not a subordinator. }
$$

This condition is naturally satisfied because the premium rate $\mu_{i}>0$ for all $i \in E$. In addition, a jump of $J$ from $i$ to $j \neq i$ has probability $q_{i j}$ of triggering a negative jump of $X$ at the same instant. We assume the law of jumps to be absolutely continuous and denote the density of the absolute value of jump size by $f_{i j}(y)$ for $y>0$ almost everywhere. We use the matrix $C$ with $\left(C_{i j}=\lambda_{i j}\left(1-q_{i j}\right)\right)_{i, j \in E}$ for transition rates without incurring jumps and the matrix $D$ with $\left(D_{i j}=\lambda_{i j} q_{i j}\right)_{i, j \in E}$ for the ones with jumps, the matrix $F=\left(f_{i j}\right)$. Note that $C+D=\Lambda$ and $q_{i i}=0$ for all $i \in E$. We further assume that the asymptotic drift of $X$ is positive, i.e. under $\mathbb{P}_{i}$ for $i \in E$,

$$
\lim _{t \rightarrow \infty} \frac{X_{t}}{t}>0 \quad \text { a.s. }
$$

which corresponds to the net profit condition in the classical ruin literature.

A matrix is said to be positive stable if all of its eigenvalues have positive real parts. For a positive stable $m \times m$ matrix $S$ and any bounded vector function $f$,

$$
\mathcal{L}\{f\}(S)=\int_{0}^{\infty} e^{-S x} f(x) \mathrm{d} x .
$$

Similarly, for any positive stable matrix $S$, the following quantities are well-defined.

$$
\psi_{S}(S)=\int_{0}^{\infty}\left(e^{-S z}-I\right) \Delta_{\nu^{S}}(d z) ; \quad \psi_{L}(S)=\int_{0}^{\infty}\left(e^{-S z}-I+S z\right) \Delta_{\nu^{z}}(d z),
$$

where $I$ is an $m \times m$ identity matrix, $\Delta_{\nu^{S}}=\operatorname{diag}\left(\nu_{1}^{S}, \cdots, \nu_{m}^{S}\right)$ and all other $\Delta$ 's are defined similarly. For brevity, we often write $\psi_{S+L}:=\psi_{S}+\psi_{L}$ and $\Delta_{\nu}=\Delta_{\nu}+\Delta_{\nu^{z}}$. The symbol * stands for the convolution, i.e., for any two conformable matrix functions $\left(A_{i k}\right)_{i \in E, k \in E^{\prime}}$ and $\left(B_{k j}\right)_{k \in E^{\prime}, j \in E^{\prime \prime}},(A * B)_{i j}=\sum_{k \in E^{\prime}} \int_{0}^{\infty} A_{i k}(x-z) B_{k j}(z) d z$. The notation $\circ$ denotes the Hadamard product (entrywise multiplication). All vectors are considered column vectors by default and $\top$ denotes the transpose operation.

According to Proposition XI.2.2 of Asmussen and Albrecher [4], we can find the following matrix moment generating function for $X_{t}$ with

$$
\mathbb{E}^{(0, i)}\left[e^{s X_{t}} ; J_{t}=j\right]=\left(e^{K(s I) t}\right)_{i j},
$$


where the matrix function $K(\cdot)$ is defined for any $m \times m$ positive stable matrix $S$,

$$
K(S):=C+S^{2} \Delta_{\sigma^{2} / 2}+S \Delta_{\mu}+\psi_{S}(S)+\psi_{L}(S)+\mathcal{L}\{D \circ F\}(S) .
$$

Lemma 2.1. The generalized Lundberg equation

$$
\operatorname{det}[K(s I)-\delta I]=0,
$$

has $m$ roots (counting multiplicity) in the region of $\mathbb{C}^{\mathrm{Re}>0}$.

Proof. Since $-X_{i}(i=1, \ldots, m)$ is not a subordinator, Theorems 1 and 2 of Ivanovs et al. [28] yield the result under the condition (2.1).

Lemma 2.2. Suppose that the equation (2.2) has $m$ distinct solutions, say $\rho_{j}(j=1, \ldots, m)$. Then there exists an $m \times m$ matrix $R$ whose eigenvalues are $\rho_{j}(j=1, \ldots, m)$ such that

$$
K(R)-\delta I=0
$$

In particular, such $R$ is positive stable and diagonalizable.

The proof can be found in Appendix B. The uniqueness of $R$ is not proven, but any such $R$ would do in the following solution representations.

\subsection{A summary of main results}

As a generalization of (1.1), we are interested in the potential (resolvent) density $r^{(\delta)}$ of the $\operatorname{MAP}(X, J)$. For a vector of measurable functions $l(x)=(l(x, 1), \cdots, l(x, m))^{\top}$, consider the following vector function

$$
H(x):=\mathbb{E}^{x}\left[\int_{0}^{\tau_{0}} e^{-\delta t} l\left(X_{t}, J_{t}\right) \mathrm{d} t\right],
$$

where $\mathbb{E}^{x}=\left(\mathbb{E}^{(x, 1)}, \cdots, \mathbb{E}^{(x, m)}\right)^{\top}$ and $\tau_{0}=\inf \left\{t: X_{t}<0\right\}$. Formally, we write

$$
H(x)=\mathbb{E}^{x}\left[\int_{0}^{\infty} e^{-\delta t} l\left(X_{t}, J_{t}\right) I\left(t<\tau_{0}\right) \mathrm{d} t\right]=\int_{0}^{\infty} \mathbb{E}^{x}\left[l\left(X_{t}, J_{t}\right) I\left(t<\tau_{0}\right)\right] \mathrm{d} t=\int_{0}^{\infty} e^{-\delta t} \mathcal{P}_{t} l(x) \mathrm{d} t,
$$

where $\left\{\mathcal{P}_{t}, t \geq 0\right\}$ is the semigroup of the MAP and determines the potential density $r^{(\delta)}$ through

$$
H(x)=\mathcal{R}_{\delta} l(x)=\int_{0}^{\infty} r^{(\delta)}(x, y) l(y) \mathrm{d} y .
$$

\section{(1) General case:}

The representation of the potential density relies on a key quantity, which is known as the $\delta$-scale matrix, say $W^{(\delta)}$, given via the Laplace transform

$$
\int_{0}^{\infty} e^{-r x} W^{(\delta)}(x) \mathrm{d} x=(K(r I)-\delta I)^{-1} .
$$

The existence of the scale matrix is shown in Ivanovs and Palmowski [29]. Note, however, one can also define the unique scale matrix using a matrix differential equation, which is equivalent to the definition via the Laplace transform (2.4) (See Section 3.3). We will show in Sections 3 and 4 that under the assumptions

$(\mathfrak{A} 1) \mathbb{E}^{(x, i)}\left[\int_{0}^{\tau_{0}} e^{-\delta t}\left|l\left(X_{t}, J_{t}\right)\right| \mathrm{d} t\right]<\infty, \quad$ for all $i \in E, x \geq 0 ;$ 
(22) $H(x)=(H(x, i))_{i \in E}$ has bounded second and third derivatives for $x \in(0, \infty)$;

$(\mathfrak{A} 3) \Delta_{\sigma^{2}}$ is non-singular;

(24) $\delta>0$ or $(\delta=0$ and $C$ is non-singular);

( $\mathfrak{A} 5)$ The equation (2.2) has $m$ distinct roots,

the resolvent density is given by

$$
r^{(\delta)}(x, y)=W^{(\delta)}(x) e^{-R y}-W^{(\delta)}(x-y), \quad x, y \geq 0,
$$

with $R$ being a positive stable and diagonalizable matrix solution to $K(R)-\delta I=0$. We learned that this result also appeared in Ivanovs [27, Theorem 1], which was derived from the use of occupation densities.

\section{(2) Particular case:}

A subclass of risk models that often appear in the ruin literature is the difference of a Brownian motion with positive drift and a subordinator, where the subordinator represents aggregate claims and the Brownian motion corresponds to fluctuations due to unexpected losses or gains. So we consider in the framework of MAP risk models the particular case where

(a6) $\int_{0}^{1} z \Delta_{\nu}(\mathrm{d} z)<\infty$.

Under assumptions $(\mathfrak{A} 1)-(\mathfrak{A} 6)$, we shall prove that the resolvent density has a more explicit form

$$
r^{(\delta)}(x, y)=\left(\int_{[0, x)} G(\mathrm{~d} u) \Delta_{2 / \sigma^{2}} B(x-u, y)\right),
$$

where the matrix function $G$ is defined in (3.12), $Q$ is a solution to the Lyaponov equation $P Q+Q R=I$ with $P=R+\Delta_{2 \mu / \sigma^{2}}$, and

$$
B(x, y)= \begin{cases}e^{-P x}\left(e^{P y} Q-Q e^{-R y}\right), & 0<y<x \\ \left(Q e^{R u}-e^{-P x} Q\right) e^{-R y}, & y>x\end{cases}
$$

The existence of $Q$ can be seen in Remark 4.1. We will also show that the scale matrix admits the series expansion

$$
W^{(\delta)}(x)=\int_{[0, x)} G(\mathrm{~d} y) \Delta_{2 / \sigma^{2}}\left(Q e^{R(x-y)}-e^{-P(x-y)} Q\right), \quad x \geq 0 .
$$

Remark 2.1. Although we did not prove (A2) in this paper, it is expected that the differentiability conditions can be proven at least in the case of (213). Similar arguments were made with jumpdiffusion models in the presence of Brownian motion in Feng [21, Appendix C].

\section{Matrix operator solution methods}

We focus on the following quantity through which the potential measure is obtained.

$$
H(x, i)=\mathbb{E}^{(x, i)}\left[\int_{0}^{\tau_{0}} e^{-\delta t} l\left(X_{t}, J_{t}\right) \mathrm{d} t\right], \quad x \geq 0,
$$

for some measurable function or generalized function $l: \mathbb{R} \times E \mapsto \mathbb{R} \cup\{\infty\}$. The quantity is interpreted as the expected present value of running costs up to ruin in Cai et al. [11]. The time of ruin can be extended to the time of default $\tau_{d}=\inf \left\{t: X_{t}<d\right\}$. However, such an extension is trivial for a spatially homogeneous model such as the MAP. Some examples of the bivariate cost function $l$ can be seen in Cheung and Feng [14], Feng [19, 20]. 


\subsection{Matrix integro-differential equation}

We first state a rather standard result for the matrix integro-differential equation satisfied by $H$. However, we are unable to pinpoint a reference to this result in the existing literature. Thus, a proof is given in Appendix B to make the paper self-contained. Interested readers may consult Jacobsen [30] for more information on the infinitesimal generator of a MAP.

Lemma 3.1. Suppose that $l(x)=(l(x, i))_{i \in E}$ is continuous in $x \in(0, \infty)$ except for a countable set of discontinuities $\mathfrak{D}$ such that $(\mathfrak{A} 1),(\mathfrak{A} 2)$ hold true. Then $H$ satisfies the following integrodifferential equation of matrix form:

$$
(\mathcal{A}-\delta) H(x)+l(x)=0,
$$

for $x \in(0, \infty) \cap \mathfrak{D}^{c}$, where $\mathcal{A}$ is the infinitesimal generator

$$
\begin{aligned}
\mathcal{A} H(x)= & \Delta_{\sigma^{2} / 2} H^{\prime \prime}(x)+\Delta_{\mu} H^{\prime}(x)+C H(x)+\int_{0}^{\infty} \Delta_{\nu^{S}}(\mathrm{~d} z)\{H(x-z)-H(x)\} \\
& +\int_{0}^{\infty} \Delta_{\nu^{Z}}(\mathrm{~d} z)\left\{H(x-z)-H(x)+z H^{\prime}(x)\right\}+(D \circ F) * H(x) .
\end{aligned}
$$

Remark 3.1. It is easy to show that since $H$ is assumed to possess bounded derivatives, it must be bounded by a polynomial, a property that is used frequently in the subsequent theorems. See, for example, Lemmas A.1 and A.2.

We seek solutions to the vector function $H$ in two cases. The first solution is based on a Markov renewal equation, which is analogous to the scalar defective renewal equation in classic ruin theory. The second solution generalizes the first solution and appears to be consistent with what would be expected from fluctuation theory. To better understand the proofs of Theorems 3.1 and 3.2, interested readers may check the scalar cases in Feng [21] and Feng and Shimizu [22].

\subsection{Solution via a Markov renewal equation}

It is shown in Feng and Shimizu [22] that a potential measure for a spectrally negative Lévy process with characteristics $\left(\mu, \sigma^{2}, \nu\right)$ can be obtained through a defective renewal equation if and only if the Levy measure $\nu$ satisfies $\int_{0}^{1} z \nu(\mathrm{d} z)<\infty$. Similarly, we can show that a potential measure for the MAP under consideration can be obtained through a Markov renewal equation if and only if (A6) is satisfied. Under this condition, the Lévy processes $\left\{Z^{(i)}, i \in E\right\}$ are restricted to Brownian motions, subordinators or their independent sums. In the case of subordinators, they can be combined with $S^{(i)}$. Hence without loss of generality, we simply consider in this subsection that $\Delta_{\nu} Z \equiv 0$, in which case $\left\{Z^{(i)}, i \in E\right\}$ reduce to Brownian motions.

Define the matrix operator $\mathcal{E}_{S}$ with a matrix function $h$ for which the integral exists

$$
\mathcal{E}_{S} h(x)=e^{-S x} \int_{0}^{x} e^{S y} h(y) \mathrm{d} y,
$$

and the matrix Dickson-Hipp operator

$$
\mathcal{T}_{S} h(x)=e^{S x} \int_{x}^{\infty} e^{-S y} h(y) \mathrm{d} y .
$$

In the case where $\Delta_{\nu}$ is a matrix of measures, it is interpreted that

$$
\mathcal{T}_{S} \Delta_{\nu}(x)=e^{S x} \int_{x}^{\infty} e^{-S y} \Delta_{\nu}(\mathrm{d} y)
$$


Theorem 3.1. Suppose that $(\mathfrak{A} 1)-(\mathfrak{A} 4)$ are true. Then $H=(H(x, i))_{i \in E}^{\top}$ satisfies the following Markov renewal equation:

$$
H(x)=(G * H)(x)+U(x), \quad x \geq 0,
$$

where

$$
G=\Delta_{\sigma^{2} / 2}^{-1} \mathcal{E}_{P}\left\{\mathcal{T}_{R} \Delta_{\nu}+\mathcal{T}_{R}(D \circ F)\right\} ; \quad U=\Delta_{\sigma^{2} / 2}^{-1} \mathcal{E}_{P} \mathcal{T}_{R} l
$$

with $R$ given in (2.3) and $P=\Delta_{2 \mu / \sigma^{2}}+R$.

Proof. We denote he entrywise differential operator $\mathcal{D}:=(\mathrm{d} / \mathrm{d} x) I$, and

$$
\begin{aligned}
H_{\nu}(x)= & \int_{0}^{\infty} \Delta_{\nu^{S}}(\mathrm{~d} z)\{H(x-z)-H(x)\} \\
& +\int_{0}^{\infty} \Delta_{\nu^{Z}}(\mathrm{~d} z)\left\{H(x-z)-H(x)+z H^{\prime}(x)\right\}+(D \circ F) * H(x) .
\end{aligned}
$$

We rewrite the equation (3.2) in terms of the operators:

$$
(B-\mathcal{D})(A+\mathcal{D}) \Delta_{\sigma^{2} / 2} H(x)=\left\{H_{\nu}+l\right\}(x), \quad x>0,
$$

where $A$ and $B$ are both $m \times m$ matrices satisfying that $A-B=\Delta_{2 \mu / \sigma^{2}}$ and $B A=(\delta I-C) \Delta_{\sigma^{2} / 2}^{-1}$. We choose $B$ to be the positive stable solution to the following quadratic matrix equation in $m \times m$ matrix $Q$.

$$
Q^{2}+Q \Delta_{2 \mu / \sigma^{2}}-(\delta I-C) \Delta_{\sigma^{2} / 2}^{-1}=0
$$

Since $C$ is a subintensity matrix, it is known that the eigenvalues of $C$ either are 0 or have negative real parts. The assumption $(\mathfrak{A} 4)$ implies that $\delta I-C$ is nonsingular and hence Lemma C.1 shows the existence and uniqueness of such a matrix $B$. We set $A:=B+\Delta_{2 \mu / \sigma^{2}}$ and henceby

$$
B A=B^{2}+B \Delta_{2 \mu / \sigma^{2}}=(\delta I-C) \Delta_{\sigma^{2} / 2}^{-1}
$$

Since $\Delta_{\sigma^{2}}$ is non-singular, we must have $H(0)=0$. Now we can use the properties (A.1) and (A.4) to obtain

$$
\Delta_{\sigma^{2} / 2} H=\mathcal{E}_{A} Y
$$

where

$$
Y=\mathcal{T}_{B}\left\{H_{\nu}+l\right\}
$$

With the matrix $R$ which is a solution to (2.3), we can show using the property (A.3) that

$$
Y=\mathcal{T}_{R}\left\{H_{\nu}+l\right\}-\mathcal{T}_{R}(B-R) \mathcal{T}_{B}\left\{H_{\nu}+l\right\}=\mathcal{T}_{R}\left\{H_{\nu}+l\right\}-\mathcal{T}_{R}(B-R) Y .
$$

We now apply property (A.5) on the second term to get

$$
Y=\mathcal{T}_{R}\left\{H_{\nu}+l\right\}-\mathcal{T}_{R}[R(B-R)+(B-R) A] \mathcal{E}_{A} Y+(B-R) \mathcal{E}_{A} Y .
$$

Since $R$ is diagonalizable and positive stable, we can expand the first term using (A.9).

$$
\begin{aligned}
Y= & \mathcal{T}_{R}\left[\psi_{S+L}(R)+\mathcal{L}\{D \circ F\}(R)\right] H+\left[\mathcal{T}_{R} \Delta_{\nu}+\mathcal{T}_{R}\{D \circ F\}\right] * H+\mathcal{T}_{R} l \\
& -\mathcal{T}_{R}[R(B-R)+(B-R) A] \mathcal{E}_{A} Y+(B-R) \mathcal{E}_{A} Y .
\end{aligned}
$$


In view of (3.7), we can simplify

$$
\mathcal{T}_{R}[R(B-R)+(B-R) A] \mathcal{E}_{A} Y=\mathcal{T}_{R}\left[(\delta I-C)-R^{2} \Delta_{\sigma^{2} / 2}-R \Delta_{\mu}\right] H .
$$

Since $R$ satisfies the matrix equation (2.3) which implies that

$$
(\delta I-C)-R^{2} \Delta_{\sigma^{2} / 2}-R \Delta_{\mu}=\psi_{S+L}(R)+\mathcal{L}\{D \circ F\}(R),
$$

we obtain from (3.9) and (3.10) that

$$
Y=\left[\mathcal{T}_{R} \Delta_{\nu}+\mathcal{T}_{R}\{D \circ F\}\right] * H+\mathcal{T}_{R} l+(B-R) \mathcal{E}_{A} Y .
$$

We choose another $m \times m$ matrix $P$ and use the property (A.2) on (3.7) to obtain

$$
\Delta_{\sigma^{2} / 2} H=\mathcal{E}_{P} Y+\mathcal{E}_{P}(P-A) \mathcal{E}_{A} Y,
$$

and that

$$
\mathcal{E}_{P} Y=\mathcal{E}_{P}\left\{\mathcal{T}_{R} \Delta_{\nu}+\mathcal{T}_{R}\{D \circ F\}\right\} * H+\mathcal{E}_{P} \mathcal{T}_{R} l+\mathcal{E}_{P}(B-R) \mathcal{E}_{A} Y
$$

Let

$$
P=A-B+R=\Delta_{\mu} \Delta_{\sigma^{2} / 2}^{-1}+R
$$

Then we shall arrive at the renewal equation.

$$
\Delta_{\sigma^{2} / 2} H=\mathcal{E}_{P}\left\{\mathcal{T}_{R} \Delta_{\nu}+\mathcal{T}_{R}\{D \circ F\}\right\} * H+\mathcal{E}_{P} \mathcal{T}_{R} l
$$

Because $\Delta_{\sigma^{2}}$ is non-singular, we obtain the Markov renewal equation (3.3).

Corollary 3.1. Suppose that $\Delta_{\sigma^{2}}=0,(\mathfrak{A} 1),(\mathfrak{A} 2),(\mathfrak{A} 4),(\mathfrak{A} 5)$ are true. Then $H=(H(x, i))_{i \in E}^{\top}$ satisfies the following Markov renewal equation:

$$
H(x)=(g * H)(x)+U(x), \quad x \geq 0,
$$

where $R$ is determined by (2.3) and

$$
g=\Delta_{\mu}^{-1}\left\{\mathcal{T}_{R} \Delta_{\nu}+\mathcal{T}_{R}(D \circ F)\right\} ; \quad U=\Delta_{\mu}^{-1} \mathcal{T}_{R} l
$$

The proof of Corollary 3.1 can be found in Appendix B.

Solving Markov renewal equations (3.3) and (3.11), we obtain the first representation of a solution to the function $H$, of a form typically seen in classical ruin theory.

Corollary 3.2. In both Theorem 3.1 and Corollary 3.1, the solution to $H$ is given by

$$
H(x)=\int_{[0, x)} G(\mathrm{~d} y) U(x-y), \quad x \geq 0,
$$

where $g$ is defined in (3.4) and

$$
G(x)=I+\sum_{k=1}^{\infty} g^{* k}(x), \quad x \geq 0 .
$$

Proof. It is proven in Appendix D that $\int_{0}^{x} g(y) \mathrm{d} y$ is a semi-Markov matrix. Thus the result follows from the Markov renewal equation (3.3) by Theorem 3.13 of Çinlar [12]. 


\subsection{Solution via the scale matrix}

Here we discuss the general case where

$$
\int_{0}^{1} z \Delta_{\nu}(\mathrm{d} z) \leq \infty
$$

Note that the Markov renewal equation approach is no longer applicable when the equality is true in (3.13).

The discussion of the existence of the scale matrix in (2.4) is provided in Ivanovs [27, Theorem 7.1] and its differentiability is provided in Kyprianou and Palmowski [34], although there are slight discrepancies in their definitions of scale matrix. For the sake of completeness, we provide some supporting arguments based on a matrix differential equation.

Here we assume $\Delta_{\sigma^{2} / 2}$ is non-degenerate. There exist a unique matrix solution $W^{(\delta)}(x)$ to the homogeneous integro-differential equation

$$
\mathcal{A} W^{(\delta)}=0,
$$

with boundary conditions

$$
W^{(\delta)^{\prime}}(0)=\Delta_{2 / \sigma^{2}}, \quad W^{(\delta)}(0)=0 .
$$

Similar boundary conditions for the scalar case can be seen in Chan et al. [13]. The proof of existence and uniqueness requires some technical but standard arguments using the Banach fixed point theorem.

We can show that $W^{(\delta)}$ has precisely the Laplace transform of what is defined as a scale matrix. Denote the first and second integrals in (3.14) by $I_{1}(x)$ and $I_{2}(x)$ respectively. Thus,

$$
\begin{aligned}
\mathcal{L} I_{1}(r) & =\int_{0}^{\infty} \Delta_{\nu^{z}}(\mathrm{~d} z)\left\{\int_{0}^{\infty} e^{-r x}\left[W^{(\delta)}(x-z)-W^{(\delta)}(x)\right] \mathrm{d} x\right\} \\
& =\int_{0}^{\infty} \Delta_{\nu^{z}}(\mathrm{~d} z)\left\{\int_{0}^{\infty} e^{-r(y+z)} W^{(\delta)}(y) \mathrm{d} y-\int_{0}^{\infty} e^{-r y} W^{(\delta)}(y) \mathrm{d} y\right\} \\
& =\int_{0}^{\infty}\left(e^{-r z}-1\right) \Delta_{\nu}(\mathrm{d} z)\left\{\int_{0}^{\infty} e^{-r y} W^{(\delta)}(y) \mathrm{d} y\right\},
\end{aligned}
$$

where we use the fact that $W^{(\delta)}(x)=0$ for $x<0$ in the penultimate equality. Similarly,

$$
\begin{aligned}
\mathcal{L} I_{2}(r) & =\int_{0}^{\infty} \Delta_{\nu^{Z}}(\mathrm{~d} z)\left\{\int_{0}^{\infty} e^{-r x}\left[W^{(\delta)}(x-z)-W^{(\delta)}(x)+z W^{(\delta)^{\prime}}(x)\right] \mathrm{d} x\right\} \\
& =\int_{0}^{\infty} \Delta_{\nu^{z}}(\mathrm{~d} z)\left\{\int_{0}^{\infty} e^{-r(y+z)} W^{(\delta)}(y) \mathrm{d} y-\int_{0}^{\infty} e^{-r y} W^{(\delta)}(y) \mathrm{d} y+r z \int_{0}^{\infty} e^{-r x} W^{(\delta)}(x) \mathrm{d} x\right\} \\
& =\int_{0}^{\infty}\left(e^{-r z}-1+r z\right) \Delta_{\nu} z(\mathrm{~d} z)\left\{\int_{0}^{\infty} e^{-r y} W^{(\delta)}(y) \mathrm{d} y\right\},
\end{aligned}
$$

where we use the boundary condition $W^{(\delta)}(0)=0$ while applying integration by parts. Thus, taking the Laplace transform on both sides of (3.14) gives

$$
(K(r I)-\delta I) \mathcal{L}\left\{W^{(\delta)}\right\}(s)-\Delta_{\sigma^{2} / 2}\left(r W^{(\delta)}(0)+W^{(\delta)^{\prime}}(0)\right)-\Delta_{\mu} W^{(\delta)}(0)=0 .
$$

Using the boundary conditions (3.15) we obtain the desired expression (2.4).

With slight abuse of notation, we define a measure $W^{(\delta)}$ by

$$
W^{(\delta)}[0, x)=W^{(\delta)}(0)+\int_{0}^{x} W^{(\delta)^{\prime}}(y) \mathrm{d} y .
$$


Whether it is a measure or a function should be clear from the context. In the case where $m>1$, $W^{(\delta)}(\mathrm{d} x)$ could be a matrix of signed measures. However the following derivations require only that the scale matrix is of bounded variation entrywise so that the Lebesgue-Stieltjes integrals are well-defined. The bounded variation follows from the almost everywhere differentiability of $W^{(\delta)}$, which is true if every $X^{(i)}$ is of unbounded variation. (See Ivanovs and Palmowski [29], the remark of Theorem 5.) In this paper, since ( $\mathfrak{A} 3)$ is assumed for simplicity, the integral with respect to a measure $W^{(\delta)}(\mathrm{d} x)$ is well-defined.

We present the second representation of a solution to the function $H$ in terms of the scale matrix, of a form typically observed in fluctuation theory. Note, however, the proof is a continuation of the matrix-operator approach used in Section 3.2.

Theorem 3.2. Suppose that $(\mathfrak{A} 1)-(\mathfrak{A} 4)$ hold true. Then it follows for all $x>0$ that

$$
H(x)=\int_{0}^{x} \Pi(\mathrm{d} y) \mathcal{T}_{R} l(x-y)
$$

where the measure $\Pi$ is defined by

$$
\Pi(\mathrm{d} x)=W^{(\delta)}(\mathrm{d} x)-W^{(\delta)}(x) R \mathrm{~d} x .
$$

Proof. Note that in this case $\Delta_{\sigma^{2} / 2}$ is non-singular and the equality (3.8) in the proof of Theorem 3.1 holds true even if $\int_{0}^{1} z \Delta_{\nu}(\mathrm{d} z)=\infty$. Taking Laplace transforms on both sides of (3.8) and using (A.8) on the first term gives for each $r>0$,

$$
\begin{aligned}
\mathcal{L} Y(r)= & (r I-R)^{-1}\left[\psi_{S+L}(R)+\mathcal{L}\{D \circ F\}(R)-\psi_{S+L}(r I)-\mathcal{L}\{D \circ F\}(r I)\right] \cdot \mathcal{L} H(r) \\
& +\mathcal{L}\left[\mathcal{T}_{R}\{N(R) H\}\right](r)+\mathcal{T}_{R} \mathcal{L}\{l\}(r) \\
& -\mathcal{L}\left\{\mathcal{T}_{R}[R(B-R)+(B-R) A] \Delta_{\sigma^{2} / 2} H\right\}(r)+(B-R) \Delta_{\sigma^{2} / 2} \mathcal{L} H(r) .
\end{aligned}
$$

We shall simplify the components of (3.19) separately. Note that

$$
\begin{aligned}
& \mathcal{L}\left[\mathcal{T}_{R}\{N(R) H\}\right](r)-\mathcal{L}\left\{\mathcal{T}_{R}[R(B-R)+(B-R) A] \Delta_{\sigma^{2} / 2} H\right\}(r) \\
= & \mathcal{L}\left[\mathcal{T}_{R}\{N(R) H\}\right](r)-\mathcal{L}\left\{\mathcal{T}_{R}\left[(\delta I-C)-R \Delta_{\mu}-R^{2} \Delta_{\sigma^{2} / 2}\right] H\right\}(r) \\
= & \mathcal{L}\left[\mathcal{T}_{R}\{(K(R)-\delta I) H\}\right](r)=0 .
\end{aligned}
$$

It is easy to verify that

$$
\mathcal{L} Y(r)=(r I+A) \Delta_{\sigma^{2} / 2} \mathcal{L} H(r) .
$$

Recall that $A-B=\Delta_{2 \mu / \sigma^{2}}$ and $B A=(\delta I-C) \Delta_{\sigma^{2} / 2}^{-1}$. Thus,

$$
\begin{aligned}
(r I-R)^{-1}\{K(r I)-\delta I\}= & \left\{(r I+A) \Delta_{\sigma^{2} / 2}-(B-R) \Delta_{\sigma^{2} / 2}\right\} \\
& -(r I-R)^{-1}\left[\psi_{S+L}(R)+\mathcal{L}\{D \circ F\}(R)-\psi_{S+L}(r I)-\mathcal{L}\{D \circ F\}(r I)\right] .
\end{aligned}
$$

Combining all the simplified components of (3.19), we obtain the following equation

$$
(r I-R)^{-1}\{K(r I)-\delta I\} \cdot \mathcal{L} H(r)=\mathcal{L}\left\{\mathcal{T}_{R} l\right\}(r),
$$

which produces (3.17) provided that the Laplace-Stieltjes transform of $\Pi$ is given by

$$
\int_{0}^{\infty} e^{-r x} \Pi(\mathrm{d} x)=[K(r I)-\delta I]^{-1}(r I-R) .
$$

We obtain the expression (3.18) using integration by parts. 


\section{Potential Measure}

As consequences of the previous analysis of solutions to $H$, we can obtain two representations of potential measures respectively under the conditions (A6) and (3.13). For notational brevity, we shall write $\mathbb{E}^{x}(Y)$ as a shorthand for $\left(\mathbb{E}^{(x, 1)}(Y), \mathbb{E}^{(x, 2)}(Y), \cdots, \mathbb{E}^{(x, m)}(Y)\right)^{\top}$.

\subsection{Series representation}

Theorem 4.1. Under the assumptions $(\mathfrak{A} 1)-(\mathfrak{A} 6)$, for any measurable function $l(x)=(l(x, i))_{i \in E}$,

$$
\mathbb{E}^{x}\left[\int_{0}^{\tau_{0}} e^{-\delta t} l\left(X_{t}, J_{t}\right) \mathrm{d} t\right]=\int_{0}^{\infty} r^{(\delta)}(x, y) l(y) \mathrm{d} y,
$$

where $r^{(\delta)}$ is given by

$$
r^{(\delta)}(x, y)=\left(\int_{[0, x)} G(\mathrm{~d} u) \Delta_{\sigma^{2} / 2}^{-1} B(x-u, y)\right),
$$

which is the density of potential measure for $X$ killed on exiting $[0, \infty)$, and $Q$ is a solution to the Lyaponov equation $P Q+Q R=I$,

$$
B(x, y)= \begin{cases}e^{-P u}\left(e^{P y} Q-Q e^{-R y}\right), & 0<y<u \\ \left(Q e^{R u}-e^{-P u} Q\right) e^{-R y}, & y>u\end{cases}
$$

Proof. Since $\Delta_{\sigma^{2}}$ is nonsingular, the point $x=0$ is regular: $\mathbb{P}\left(\tau_{0}=0\right)=1$. Hence, $H(0)=0$ when $l$ is measurable, and it follows from Corollary 3.2 that

$$
H(x)=\int_{[0, x)} G(\mathrm{~d} y) \Delta_{\sigma^{2} / 2}^{-1} \mathcal{E}_{P} \mathcal{T}_{R} l(x-y), \quad x \geq 0 .
$$

Note that $P Q+Q R=I$. Using the identity (A.6), we must have

$$
\begin{aligned}
\mathcal{E}_{P} \mathcal{T}_{R} l(x)= & e^{-P x} \int_{0}^{x}\left(e^{P y} Q-Q e^{-R y}\right) l(y) \mathrm{d} y \\
& +\int_{x}^{\infty}\left(Q e^{R x}-e^{-P x} Q\right) e^{-R y} l(y) \mathrm{d} y=\int_{0}^{\infty} B(x, y) l(y) \mathrm{d} y .
\end{aligned}
$$

Changing the order of integration yields the desired solution.

Remark 4.1. According to Theorem 4.4.6 of Horn and Johnson [26, p270], the equation PQ+ $Q R=I$ has a unique solution $Q$ if and only if $P$ and $R$ have no common eigenvalues. This is clearly the case when $\Delta_{2 \mu / \sigma^{2}} \neq 0$. In this case, the vector of stacked columns of $Q$ denoted by $\operatorname{vec} Q$ is determined by

$$
\operatorname{vec} Q=\left(I \otimes P+R^{\top} \otimes I\right)^{-1} \operatorname{vec} I,
$$

where $\otimes$ denotes the Kronecker product.

Interestingly, the Lyaponov equation seems to play an important role in the representations of many exit problems for MAPs. A similar Lyaponov equation appears in Albrecher and Ivanovs [3] survival probability of a MAP risk model with Poissonian observations. 


\subsection{Scale matrix representation}

Theorem 4.2. Under the assumptions $(\mathfrak{A} 1)-(\mathfrak{A} 5)$, for any measurable function $l(x)=(l(x, i))_{i \in E}$,

$$
\mathbb{E}^{x}\left[\int_{0}^{\tau_{0}} e^{-\delta t} l\left(X_{t}, J_{t}\right) \mathrm{d} t\right]=\int_{0}^{\infty} r^{(\delta)}(x, y) l(y) \mathrm{d} y,
$$

where $r^{(\delta)}(x, y)=W^{(\delta)}(x) e^{-R y}-W^{(\delta)}(x-y)$, for $x, y \geq 0$, which is the density of the potential measure for $X$ killed on exiting $[0, \infty)$.

Proof. We first assume that all assumptions of Theorem 3.2 are satisfied. Since $\Delta_{\sigma^{2}}$ is nonsingular, we must have $H(0)=0$. Hence,

$$
\begin{aligned}
H(x)= & \int_{0}^{x} \Pi(\mathrm{d} y) \mathcal{T}_{R} l(x-y) \\
= & \int_{0}^{x}\left[W^{(\delta)}(\mathrm{d} y)-W^{(\delta)}(y) R \mathrm{~d} y\right] e^{R(x-y)} \int_{x-y}^{\infty} e^{-R z} l(z) \mathrm{d} z \\
= & \int_{0}^{x}\left(\int_{x-z}^{x} W^{(\delta)}(\mathrm{d} y) e^{-R y}-W^{(\delta)}(y) R e^{-R y} \mathrm{~d} y\right) e^{R(x-z)} l(z) \mathrm{d} z \\
& +\int_{x}^{\infty}\left(\int_{0}^{x} W^{(\delta)}(\mathrm{d} y) e^{-R y}-W^{(\delta)}(y) R e^{-R y} \mathrm{~d} y\right) e^{R(x-z)} l(z) \mathrm{d} z .
\end{aligned}
$$

Note that $W^{(\delta)}(\mathrm{d} y)=W^{(\delta)^{\prime}}(y) \mathrm{d} y$ on $(x-z, x)$. Thus, using integration by parts, we obtain

$$
\begin{aligned}
& \int_{x-z}^{x} W^{(\delta)}(\mathrm{d} y) e^{-R y}-W^{(\delta)}(y) R e^{-R y} \mathrm{~d} y \\
= & \int_{x-z}^{x} W^{(\delta)}(\mathrm{d} y) e^{-R y}+W^{(\delta)}(x) e^{-R x}-W^{(\delta)}(x-z) e^{-R(x-z)}-\int_{x-z}^{x} \mathrm{~d} W^{(\delta)}(y) e^{-R y} \\
= & W^{(\delta)}(x) e^{-R x}-W^{(\delta)}(x-z) e^{-R(x-z)} .
\end{aligned}
$$

Similarly, because of (3.16), we have

$$
\begin{aligned}
& \int_{0}^{x} W^{(\delta)}(\mathrm{d} y) e^{-R y}-W^{(\delta)}(y) R e^{-R y} \mathrm{~d} y \\
= & \int_{0}^{x} W^{(\delta)}(\mathrm{d} y) e^{-R y}+W^{(\delta)}(x) e^{-R x}-W^{(\delta)}(0)-\int_{0}^{x} \mathrm{~d} W^{(\delta)}(y) e^{-R y}=W^{(\delta)}(x) e^{-R x} .
\end{aligned}
$$

Using the fact that $W^{(\delta)}(z)=0$ when $z<0$, we have proved the desired expression.

The two representations of the resolvent density for a spectrally negative MAP are generalizations of those for a spectrally negative Lévy process shown in the introduction. The next theorem establishes the equivalence of the two representations.

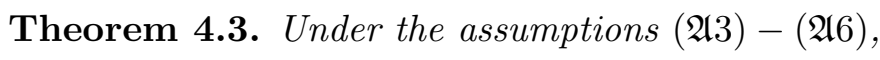

$$
W^{(\delta)}(x)=\int_{[0, x)} G(\mathrm{~d} y) \Delta_{\sigma^{2} / 2}^{-1}\left(Q e^{R(x-y)}-e^{-P(x-y)} Q\right), \quad x \geq 0 .
$$

Proof. Denote the expression on the right-hand side by RHS and take Laplace transform.

$$
\begin{aligned}
\mathcal{L}\{\operatorname{RHS}\}(r) & =\int_{[0, \infty)}^{\infty} e^{-r y} G(\mathrm{~d} y) \Delta_{\sigma^{2} / 2}^{-1}\left[Q(r I-R)^{-1}-(r I+P)^{-1} Q\right] \\
& =\int_{[0, \infty)}^{\infty} e^{-r y} G(\mathrm{~d} y) \Delta_{\sigma^{2} / 2}^{-1}(r I+P)^{-1}(r I-R)^{-1}
\end{aligned}
$$


with the last equality from the fact that $P Q+Q R=I$. It is easy to show that

$$
\int_{0}^{\infty} e^{-r y} \mathcal{T}_{R} \Delta_{\nu}(y) \mathrm{d} y=(R-r I)^{-1}\left[\psi_{S}(r I)-\psi_{S}(R)\right] .
$$

Thus, we obtain

$$
\begin{aligned}
& \int_{[0, \infty)} e^{-r y} G(\mathrm{~d} y)=\left(I-\int_{[0, \infty)} e^{-r y} G(y) \mathrm{d} y\right)^{-1} \\
= & \left(I-\Delta_{\sigma^{2} / 2}^{-1}(P+r I)^{-1}(R-r I)^{-1}\left[\psi_{S}(r I)-\psi_{S}(R)+\mathcal{L}\{D \circ F\}(r I)-\mathcal{L}\{D \circ F\}(R)\right]\right)^{-1} \\
= & (\delta I-K(r I))^{-1}(R-r I)(P+r I) \Delta_{\sigma^{2} / 2},
\end{aligned}
$$

since

$$
\begin{aligned}
& (R-r I)(P+r I) \Delta_{\sigma^{2} / 2}-\left[\psi_{S}(r I)-\psi_{S}(R)+\mathcal{L}\{D \circ F\}(r I)-\mathcal{L}\{D \circ F\}(R)\right] \\
& =\left[R^{2} \Delta_{\sigma^{2} / 2}+R \Delta_{\mu}+\psi_{S}(R)+\mathcal{L}\{D \circ F\}(R)+C\right]-\left[r^{2} \Delta_{\sigma^{2} / 2}+r \Delta_{\mu}+\psi_{S}(r I)+\mathcal{L}\{D \circ F\}(r I)+C\right] \\
& =\delta I-K(r I) .
\end{aligned}
$$

Therefore, simple substitutions yield $\mathcal{L}\{\mathrm{RHS}\}=\mathcal{L}\left\{W^{(\delta)}\right\}=(K(r I)-\delta I)^{-1}$, which confirms the equality as the scale matrix is uniquely determined by its Laplace transform.

\section{Applications}

Quantities of interest in ruin theory are often functionals of the underlying risk process, many of which are special cases of the function $H$. By identifying the corresponding $l$, we can obtain explicit solutions to all such functionals by Theorems 4.1 and 4.2 .

\subsection{Total claim costs up to ruin}

The expected present value (EPV) of penalty at ruin, known as the Gerber-Shiu function, is the classical tool used to analyze the joint distribution of the surplus prior to ruin, the deficit at ruin as well as the time of ruin. It is known in many risk models driven by Markov processes that the Gerber-Shiu function can be retrieved from the EPV of total claim costs up to ruin, which by itself is a special case of function $H$. We can easily draw a similar connection among these quantities in the context of Markov additive processes.

A natural extension of the EPV of total claim costs up to ruin in the Markov additive risk model is given by

$$
M(x)=\mathbb{E}^{x}\left[\sum_{0 \leq t \leq \tau_{0}} e^{-\delta t} \varpi_{\left(J_{t-}, J_{t}\right)}\left(X_{t-}, X_{t}\right) I\left(\left|\Delta X_{t}\right|>0\right)\right],
$$

where $\varpi_{(i, j)}(x, y)$ takes into account the cost of every claim depending on the state of MAP $(x, i)$ prior to the claim and the state $(y, j)$ immediately following the claim. We write $\Delta_{\varpi}(x, y)=$ $\operatorname{diag}\left(\varpi_{(i, i)}(x, y)\right)_{i \in E}$ and $\varpi=\left(\varpi_{(i, j)}(x, y)\right)_{i, j \in E}$.

Proposition 5.1. $M$ can be obtained from $H$ by letting

$$
l(x)=\left\{\int_{0}^{\infty} \Delta_{\varpi}(x, x-z) \Delta_{\nu}(\mathrm{d} z)+\int_{0}^{\infty} D \circ \varpi(x, x-z) \circ F(z) \mathrm{d} z\right\} \mathbf{1} .
$$


Proof. Consider the case $J_{0}=i$. Let $T_{1}=\inf \left\{t: J_{t} \neq i\right\}$. Using the strong Markov property, we obtain

$$
\begin{aligned}
M_{i}(x)= & \mathbb{E}^{(x, i)}\left[\sum_{0 \leq t<T_{1}} e^{-\delta t} \varpi_{\left(J_{t-}, J_{t}\right)}\left(X_{t-}, X_{t}\right) I\left(\Delta X_{t}>0\right)\right. \\
& \left.+e^{-\delta T_{1}} \varpi_{\left(J_{T_{1}-}, J_{T_{1}}\right)}\left(X_{T_{1}-}, X_{T_{1}}\right) I\left(\Delta X_{T_{1}}>0\right)+e^{-\delta T_{1}} M_{J_{T_{1}}}\left(X_{T_{1}}\right)\right] .
\end{aligned}
$$

Note that $X_{t}=X_{t}^{(i)}$ and $J_{t}=i$ for $0 \leq t<T_{1}$. Thus,

$$
\begin{aligned}
& \mathbb{E}^{(x, i)}\left[\sum_{0 \leq t<T_{1}} e^{-\delta t} \varpi_{\left(J_{t-}, J_{t}\right)}\left(X_{t-}, X_{t}\right) I\left(\Delta X_{t}>0\right)\right] \\
& =\mathbb{E}^{(x, i)}\left[\int_{0}^{T_{1}} e^{-\delta t} \int_{0}^{\infty} \varpi_{(i, i)}\left(X_{t}, X_{t}-z\right) \nu_{i}^{S+Z}(\mathrm{~d} z) \mathrm{d} t\right],
\end{aligned}
$$

where we used the compensation formula (cf. Section 4.3.2 of Kyprianou [32] or Bertoin [6], page 7). Similarly, we can show

$$
\begin{aligned}
& \mathbb{E}^{(x, i)}\left[e^{-\delta T_{1}} \varpi_{\left(J_{T_{1}-}, J_{T_{1}}\right)}\left(X_{T_{1}-}, X_{T_{1}}\right) I\left(\Delta X_{T_{1}}>0\right)\right] \\
= & \mathbb{E}^{(x, i)}\left[\int_{0}^{T_{1}} e^{-\delta t} \sum_{j \in E} \lambda_{i j} q_{i j} \int_{0}^{\infty} \varpi_{(i, j)}\left(X_{t}, X_{t}-z\right) f_{i j}(z) \mathrm{d} z \mathrm{~d} t\right] .
\end{aligned}
$$

In view of (5.2), (5.3) and (5.4), we obtain

$$
\begin{aligned}
& M_{i}(x)=\mathbb{E}^{(x, i)}\left[e^{-\delta T_{1}} M_{J_{T_{1}}}\left(X_{T_{1}}\right)\right] \\
& +\mathbb{E}^{(x, i)}\left[\int_{0}^{T_{1}} e^{-\delta t}\left\{\int_{0}^{\infty} \varpi_{(i, i)}\left(X_{t}, X_{t}-z\right) \nu_{i}^{S+Z}(\mathrm{~d} z)+\sum_{j \in E} \lambda_{i j} q_{i j} \int_{0}^{\infty} \varpi_{(i, j)}\left(X_{t}, X_{t}-z\right) f_{i j}(z) \mathrm{d} z\right\} \mathrm{d} t\right] .
\end{aligned}
$$

Applying the strong Markov property to $H(x, i)$, we also obtain

$$
H(x, i)=\mathbb{E}^{(x, i)}\left[\int_{0}^{T_{1}} e^{-\delta t} l\left(X_{t}\right) \mathrm{d} t\right]+\mathbb{E}^{(x, i)}\left[e^{-\delta T_{1}} H\left(X_{T_{1}}, J_{T_{1}}\right)\right] .
$$

It follows immediately from (5.5) and (5.6) that $M$ is a special case of $H$ with (5.1).

Remark 5.1. Similar arguments show that the following quantities are all special cases of $H$.

1. Expected present value (EPV) of total costs due to regime changes (a transition in the Markov chain $J): M^{C}(x)=\left(M_{1}^{C}(x), \cdots, M_{m}^{C}(x)\right)^{\top}$ where

$$
M_{i}^{C}(x)=\mathbb{E}^{(x, i)}\left[\sum_{0 \leq t \leq \tau_{0}} e^{-\delta t} \varpi_{\left(J_{t-}, J_{t}\right)}\left(X_{t-}, X_{t}\right) I\left(\left|\Delta X_{t}\right|>0, J_{t-} \neq J_{t}\right)\right],
$$

corresponds to the cost function

$$
l(x)=\left\{\int_{0}^{\infty} D \circ \varpi(x, x-z) \circ F(z) \mathrm{d} z\right\} \mathbf{1} ;
$$


2. EPV of total costs due to jumps caused by the subordinator within each regime: $M^{D}(x)=$ $\left(M_{1}^{D}(x), \cdots, M_{m}^{D}(x)\right)^{\top}$ where

$$
M_{i}^{D}(x)=\mathbb{E}^{(x, i)}\left[\sum_{0 \leq t \leq \tau_{0}} e^{-\delta t} \varpi_{\left(J_{t-}, J_{t}\right)}\left(X_{t-}, X_{t}\right) I\left(\left|\Delta X_{t}\right|=\Delta S_{J_{t}}(t)>0, J_{t-}=J_{t}\right)\right],
$$

corresponds to the cost function

$$
l(x)=\left\{\int_{0}^{\infty} \Delta_{\varpi}(x, x-z) \Delta_{\nu S}(\mathrm{~d} z)\right\} \mathbf{1}
$$

3. EPV of total costs due to jumps caused by the SPL process within each regime: $M^{D}(x)=$ $\left(M_{1}^{D}(x), \cdots, M_{m}^{D}(x)\right)^{\top}$ where

$$
M_{i}^{D}(x)=\mathbb{E}^{(x, i)}\left[\sum_{0 \leq t \leq \tau_{0}} e^{-\delta t} \varpi_{\left(J_{t-}, J_{t}\right)}\left(X_{t-}, X_{t}\right) I\left(\left|\Delta X_{t}\right|=\Delta Z_{J_{t}}(t)>0, J_{t-}=J_{t}\right)\right],
$$

corresponds to the cost function

$$
l(x)=\left\{\int_{0}^{\infty} \Delta_{\varpi}(x, x-z) \Delta_{\nu}(\mathrm{d} z)\right\} \mathbf{1}
$$

4. The Gerber-Shiu function: $m_{\cdot j}(x)=\left(m_{1 j}(x), \cdots, m_{m j}(x)\right)^{\top}$ for any $j \in E$, where

$$
m_{i j}(x)=\mathbb{E}^{(x, i)}\left[e^{-\delta \tau_{0}} w_{\left(J_{\tau_{0}-}, J_{\tau_{0}}\right)}\left(X_{\tau_{0}-},\left|X_{\tau_{0}}\right|\right) I\left(\tau_{0}<\infty, J_{\tau_{0}}=j\right)\right],
$$

corresponds to the cost function

$$
\begin{aligned}
l(x)= & \left(\int_{x}^{\infty} \Delta_{w}(x, z-x) \Delta_{\nu}(\mathrm{d} z)\right)_{\cdot j} \\
& +\left(\int_{x}^{\infty} D \circ w(x, z-x) \circ F(z) \mathrm{d} z\right)_{\cdot j}+\left(w(0,0) \delta_{0}(x)\right)_{\cdot j},
\end{aligned}
$$

where $\Delta_{w}(x, y)=\operatorname{diag}\left(w_{(i, i)}(x, y)\right)_{i \in E}$ and $w(x, y)=\left(w_{(i, j)}(x, y)\right)_{i, j \in E}$.

Remark 5.2. In the case where $w(0,0)=0$, we apply Corollary 4.2 with the cost function (5.7) to obtain the solution to the Gerber-Shiu function $m(x)=\left(m_{i j}(x)\right)_{i, j \in E}$

$$
m(x)=\int_{0}^{\infty} \int_{0}^{\infty} R^{(\delta)}(x, y)\left[\Delta_{w}(y, u) \Delta_{\nu}(\mathrm{d} u+y)+D \circ w(y, u) \circ F(u+y) \mathrm{d} u\right] \mathrm{d} y .
$$

This formula is a generalization of Theorem 4.1 of Asmussen and Albrecher[4, p.385].

\subsection{Total operating costs up to ruin with ruin occurring}

In many papers on MAP risk models, the state of the Markov chain $J$ at the time of ruin $\tau_{0}$ is specified in the definition of functionals. In doing so, they implicitly assume that $\tau_{0}<\infty$, which is restricted to the cases where ruin does occur. Here we want to point out that such functionals are also special cases of $H$. For example, define $U(x)=\left(U_{i j}(x)\right)_{i, j \in E}$ where

$$
U_{i j}(x)=\mathbb{E}^{(x, i)}\left[\left(\int_{0}^{\tau_{0}} e^{-\delta t} l_{J_{t}}^{*}\left(X_{t}\right) \mathrm{d} t\right) I\left(\tau_{0}<\infty, J_{\tau_{0}}=j\right)\right] .
$$


Proposition 5.2. $U$ can be obtained from $H$ by letting $l=\left(l^{*} \circ L_{\cdot j}\right)_{j \in E}$, where

$$
L(x)=\int_{0}^{\infty} \int_{0}^{\infty} R^{(0)}(x, y)\left[\Delta_{\nu}(\mathrm{d} u+y)+D \circ F(u+y) \mathrm{d} u\right] \mathrm{d} y .
$$

Proof. We can use the strong Markov property to represent $U$ in the form of $H$.

$$
\begin{aligned}
U_{i j}(x) & =\mathbb{E}^{(x, i)}\left[\left(\int_{0}^{\infty} e^{-\delta t} l_{J_{t}}^{*}\left(X_{t}\right) I_{\left\{X_{s} \geq 0 \forall 0 \leq s \leq t\right\}} I_{\left\{\tau_{0}<\infty, J_{\tau_{0}}=j\right\}} \mathrm{d} t\right)\right] \\
& =\mathbb{E}^{(x, i)}\left[\int_{0}^{\infty} \mathbb{E}^{(x, i)}\left\{e^{-\delta t} l_{J_{t}}^{*}\left(X_{t}\right) I_{\left\{X_{s} \geq 0 \forall 0 \leq s \leq t\right\}} I_{\left\{\tau_{0}<\infty, J_{\tau_{0}}=j\right\}} \mid \mathcal{F}_{t}\right\} \mathrm{d} t\right] \\
& =\mathbb{E}^{(x, i)}\left[\int_{0}^{\infty} e^{-\delta t} l_{J_{t}}^{*}\left(X_{t}\right) I_{\left\{X_{s} \geq 0 \forall 0 \leq s \leq t\right\}} \mathbb{E}^{(x, i)}\left\{I_{\left\{\tau_{0}<\infty, J_{\tau_{0}}=j\right\}} \mid \mathcal{F}_{t}\right\} \mathrm{d} t\right] \\
& =\mathbb{E}^{(x, i)}\left[\int_{0}^{\tau_{0}} e^{-\delta t} l_{J_{t}}^{*}\left(X_{t}\right) P_{J_{t}, j}\left(X_{t}\right) \mathrm{d} t\right],
\end{aligned}
$$

where $L_{i j}(x)=\mathbb{P}^{(x, i)}\left\{\tau<\infty, J_{\tau}=j\right\}$ is the ruin probability. The expression (5.8) follows from Remark 5.2 by letting $w(x, y)=1$, which is a matrix of all 1 's.

\subsection{Connections to exit problems}

Kyprianou and Palmowski [34] recently established many identities regarding exit problems of MAPs. The paper employs time reversal arguments and the Asmussen-Kella martingale, with an analogy to a similar technical treatment for a spectrally negative Levy process in Kyprianou and Palmowski [33]. As by-products of results from Section 5.2, we are able to reaffirm some of their identities. Although not as comprehensive as the approach of Kyprianou and Palmowski [34], our approach is based merely on elementary matrix operator identities. It should also be noted that no assumption on the stationary distribution of $J$ is made in this paper, whereas the time reversal arguments in Kyprianou and Palmowski [34] required $J_{0}$ to have stationary distribution. This matrix operator approach avoids the stationary distribution assumption so that delayed risk models in which $J_{0}$ has an arbitrary distribution can be analyzed in the same framework.

First, we provide a result for the Laplace transform of the time of ruin (interpreted as the discounted probability of ruin) defined by $V(x)=\left(V_{i j}\right)_{i, j \in E}$, where

$$
V_{i j}(x)=\mathbb{E}^{(x, i)}\left[e^{-\delta \tau_{0}} I\left(\tau_{0}<\infty, J_{\tau_{0}}=j\right)\right] .
$$

Theorem 5.1. The Laplace-Stieltjes transform of $v$ is given by

$$
\int_{0}^{\infty} e^{-r x} \mathrm{~d} V(x)=(K(r)-\delta I)^{-1}\left(I-r R^{-1}\right)(\delta I-\Lambda) .
$$

Proof. Clearly, the function $V_{i j}$ can be retrieved from the Gerber-Shiu function $m_{i j}$ by letting $w_{i j}(x, y)=1$. In view of $(3.20), \mathcal{L} V(r)=\int_{0}^{\infty} e^{-r y} V(y) \mathrm{d} y$ must satisfy

$$
(r I-R)^{-1}\{K(r I)-\delta I\} \cdot \mathcal{L} V(r)=\mathcal{L}\left\{\mathcal{T}_{R} A\right\}(r)+\Delta_{\sigma^{2} / 2} V(0),
$$

where $V(0)=I$ and

$$
A(x):=A_{1}(x)+A_{2}(x)+A_{3}(x):=\int_{x}^{\infty} \Delta_{\nu}(\mathrm{d} z)+\int_{x}^{\infty} D \circ F(z) \mathrm{d} z+\delta_{0}(x) I .
$$

Because of (A.3), we obtain

$$
\{K(r I)-\delta I\} \cdot \mathcal{L} V(r)=\mathcal{L} A(R)-\mathcal{L} A(r I)+(r I-R) \Delta_{\sigma^{2} / 2} .
$$


We claim that

$\mathcal{L} A(R)-\mathcal{L} A(r I)=(r R)^{-1}\left[R\left(\psi_{S+L}(r I)+\mathcal{L}\{D \circ F\}(r I)-D\right)-r\left(\psi_{S+L}(R)+\mathcal{L}\{D \circ F\}(R)-D\right)\right]$

It follows that

$$
\begin{aligned}
& \mathcal{L} A(R)-\mathcal{L} A(r I)+(r I-R) \frac{1}{2} \Delta_{\sigma^{2}} \\
= & (r R)^{-1}\left[R\left(r \Delta_{\mu}+\psi_{S+L}(r I)+\mathcal{L}\{D \circ F\}(r I)-D\right)-r\left(R \Delta_{\mu}+\psi_{S+L}(R)+\mathcal{L}\{D \circ F\}(R)-D\right)\right] \\
+ & (r R)^{-1}\left(\frac{1}{2} R r^{2} \Delta_{\sigma^{2}}-\frac{1}{2} r R^{2} \Delta_{\sigma^{2}}\right) \\
= & (r R)^{-1}[R K(r)-R(C+D)-r K(R)+r(C+D)]=(r R)^{-1}[R K(r)-R \Lambda-r K(R)+r \Lambda] .
\end{aligned}
$$

Therefore, it follows from (B.6) that

$$
\mathcal{L} V(r)=(K(r)-\delta I)^{-1}(r R)^{-1}[R K(r)-R \Lambda-r K(R)+r \Lambda]
$$

In other words,

$$
\begin{aligned}
\int_{0}^{\infty} e^{-r x} \mathrm{~d} V(x) & =r \int_{0}^{\infty} e^{-r x} V(x) \mathrm{d} x-V(0) \\
& =(K(r)-\delta I)^{-1} R^{-1}[R K(r)-R \Lambda-r K(R)+r \Lambda]-I \\
& =(K(r)-\delta I)^{-1} R^{-1}[\delta R-R \Lambda-r K(R)+r \Lambda] .
\end{aligned}
$$

which leads to (5.9) after rearrangement.

Last, we give a proof of (5.11). For any positive stable matrix $S$, we have that

$$
\mathcal{L} A_{2}(S)=\int_{0}^{\infty} \int_{x}^{z} e^{-S x} D \circ F(\mathrm{~d} z)=\int_{0}^{\infty} S^{-1}\left(I-e^{-S z}\right) D \circ F(\mathrm{~d} z)=S^{-1}[D-\mathcal{L}\{D \circ F\}(S)]
$$

Hence,

$$
\mathcal{L} A_{2}(R)-\mathcal{L} A_{2}(r I)=(R r)^{-1}\left[R\left(\int_{0}^{\infty} e^{-r z} D \circ F(\mathrm{~d} z)-D\right)-r\left(\int_{0}^{\infty} e^{-R z} D \circ F(\mathrm{~d} z)-D\right)\right]
$$

For any positive stable matrix $S$ and $\epsilon>0$, we also have that

$$
\begin{aligned}
\int_{\epsilon}^{\infty} e^{-S x} \int_{x}^{\infty} \Delta_{\nu}(\mathrm{d} z) \mathrm{d} x & =\int_{\epsilon}^{\infty} \int_{\epsilon}^{z} e^{-S x} \mathrm{~d} x \Delta_{\nu}(\mathrm{d} z) \\
& =S^{-1} e^{-\epsilon S} \int_{\epsilon}^{\infty} \Delta_{\nu}(\mathrm{d} z)-S^{-1} \int_{\epsilon}^{\infty} e^{-S z} \Delta_{\nu}(\mathrm{d} z)
\end{aligned}
$$


Note that $\nu_{i}^{S+L}$ is a finite measure on $[\epsilon, \infty)$ for all $i \in E$. Define $\tilde{l}_{1}^{\epsilon}(r):=\int_{\epsilon}^{\infty} e^{-r x} l_{1}(x) \mathrm{d} x$. Then

$$
\begin{aligned}
\tilde{l}_{1}^{\epsilon}(R)-\tilde{l}_{1}^{\epsilon}(r I)= & (R r)^{-1}\left[r e^{-R \epsilon} \int_{\epsilon}^{\infty} \Delta_{\nu}(\mathrm{d} z)-R e^{-r \epsilon} \int_{\epsilon}^{\infty} \Delta_{\nu}(\mathrm{d} z)\right. \\
& \left.+R \int_{\epsilon}^{\infty} e^{-r z} \Delta_{\nu}(\mathrm{d} z)-r \int_{\epsilon}^{\infty} e^{-R z} \Delta_{\nu}(\mathrm{d} z)\right] \\
& \left.+R \int_{\epsilon}^{\infty}\left(e^{-r z}+r z-1\right) \Delta_{\nu}(\mathrm{d} z)-r \int_{\epsilon}^{\infty}\left(e^{-R z}+R z-I\right) \Delta_{\nu}(\mathrm{d} z)\right] \\
= & (R r)^{-1}\left[r\left(e^{-R \epsilon}+R \epsilon-I\right) \int_{\epsilon}^{\infty} \Delta_{\nu}(\mathrm{d} z)-R\left(e^{-r \epsilon}+r \epsilon-1\right) \int_{\epsilon}^{\infty} \Delta_{\nu}(\mathrm{d} z)\right. \\
& \left.+R \int_{\epsilon}^{\infty}\left(e^{-r z}+r z-1\right) \Delta_{\nu}(\mathrm{d} z)-r \int_{\epsilon}^{\infty}\left(e^{-R z}+R z-I\right) \Delta_{\nu}(\mathrm{d} z)\right] .
\end{aligned}
$$

Noticing that $\left|\epsilon^{2} I(z \geq \epsilon)\right| \leq z^{2}$ and that $\int_{0}^{1} z^{2} \nu_{S+L}(\mathrm{~d} z)<\infty$, we see that

$$
\left(e^{-r \epsilon}+r \epsilon-1\right) \int_{\epsilon}^{\infty} \Delta_{\nu^{S}}(\mathrm{~d} z) \leq \frac{r^{2}}{2} \int_{0}^{1} \epsilon^{2} I(z \geq \epsilon) \Delta_{\nu}(\mathrm{d} z) \rightarrow 0, \quad \epsilon \rightarrow 0,
$$

by the Lebesgue convergence theorem, which yields (5.11).

Secondly, we can also produce a result regarding the running infimum of the process denoted by $\underline{X}_{t}:=\inf _{0 \leq s \leq t} X_{s}$. Define the matrix function $O$ by

$$
O_{i j}(s)=\mathbb{E}^{(0, i)}\left[e^{s \underline{X}_{e_{\delta}}} I\left(J_{e_{\delta}}=j\right)\right] .
$$

Corollary 5.1. The solution to $O$ is given by

$$
O(s)=\delta(K(s)-\delta I)^{-1}\left(s R^{-1}-I\right)
$$

Proof. Let $e_{\delta}$ be an independent exponential random variable with mean $1 / \delta$. Note that

$$
\begin{aligned}
\mathbb{P}^{(x, i)}\left(\tau_{0}<e_{\delta}, J_{e_{\delta}}=j\right) & =\mathbb{E}^{(x, i)}\left[I\left(\tau_{0}<e_{\delta}\right) \mathbb{E}^{\left(X_{\tau_{0}}, J_{\tau_{0}}\right)}\left\{I\left(J_{e_{\delta}-\tau_{0}}=j\right)\right\}\right] \\
& =\sum_{k \in E} \mathbb{P}^{(x, i)}\left(\tau_{0}<e_{\delta}, J_{\tau_{0}}=k\right) \mathbb{P}\left\{J_{0}=k, J_{e_{\delta}}=j\right\}
\end{aligned}
$$

with last equality from the memoryless property of $e_{\delta}$ and that $J$ is independent of $X$.

Since $\mathbb{E}^{(x, i)}\left[e^{-\delta \tau_{0}} I\left(\tau_{0}<\infty, J_{\tau_{0}}=j\right)\right]=\mathbb{P}^{(x, i)}\left(\tau_{0}<e_{\delta}, J_{\tau_{0}}=j\right)$, we see that

$$
\begin{aligned}
& \mathbb{P}^{(0, i)}\left(-\underline{X}_{e_{\delta}}>x, J_{e_{\delta}}=j\right)=\mathbb{P}^{(x, i)}\left(\tau_{0}<e_{\delta}, J_{e_{\delta}}=j\right) \\
= & \sum_{k \in E} \mathbb{E}^{(x, i)}\left[e^{-\delta \tau_{0}} I\left(\tau_{0}<\infty, J_{\tau_{0}}=k\right)\right] \mathbb{P}\left\{J_{0}=k, J_{e_{\delta}}=j\right\}=\delta \sum_{k \in E} v_{i k}(x)(\delta I-\Lambda)_{k j}^{-1} .
\end{aligned}
$$

Note that $O_{i j}(x)=\int_{0}^{\infty} e^{-s x} \mathrm{~d} \mathbb{P}^{(0, i)}\left(-\underline{X}_{e_{\delta}} \leq x, J_{e_{\delta}}=j\right)$. Hence, the desired result follows from $O(x)=-\int_{0}^{\infty} e^{-s x} \mathrm{~d} V(x)\left\{\delta(\delta I-\Lambda)^{-1}\right\}$.

Remark 5.3. The formulas (5.9) and (5.12) are in exact agreement with equation (7), (9) of Kyprianou and Palmowski [34]. Note that (9) was derived from (7) in Kyprianou and Palmowski [34] using time-reversal and Asmussen-Kella martingale arguments but our proof is much simpler. A quick comparison shows that the matrix $\hat{D}(q)^{\top}$ (they used $q$ in place of $\delta$ ) in their paper is effectively the matrix $R^{-1}$ in this paper. It is worthwhile to note that Kyprianou and Palmowski [34] stated (p.125) "establishing an expression of $\Lambda(q)$ for spectrally negative MAPs is an open question". This problem has been addressed in Ivanovs and Palmowski [29] but here we provide an alternative derivation using integral and differential operators. 


\section{Acknowledgement}

The authors would like to thank Dr. Jevgenijs Ivanovs for bringing to our attention his recent work [27] on the subject. The invaluable suggestions from Dr. Ivanovs and the anonymous referee for improving the paper are greatly appreciated. This research has been partially supported by the Ministry of Education, Science, Sports and Culture, Grant-in-Aid for Young Scientists (B), no.24740061, 2013-2014, and Japan Science and Technology Agency, CREST.

\section{A Matrix identities}

For brevity, we often use $e^{-S \cdot}$ to indicate the function $x \mapsto e^{-S x}$. We suppress the argument $x$ whenever it is clear from context.

Lemma A.1. For any matrices $S_{1}, S_{2}$, and vector function $h$,

$$
\begin{aligned}
\mathcal{E}_{S_{1}}\left(S_{1}+\mathcal{D}\right) h & =H-e^{-S_{1} \cdot h(0) ;} \\
\mathcal{E}_{S_{1}}\left(S_{1}-S_{2}\right) \mathcal{E}_{S_{2}} h & =\mathcal{E}_{S_{2}} H-\mathcal{E}_{S_{1}} h ; \\
\mathcal{T}_{S_{1}}\left(S_{2}-S_{1}\right) \mathcal{T}_{S_{2}} h & =\mathcal{T}_{S_{1}} H-\mathcal{T}_{S_{2}} h,
\end{aligned}
$$

provided that $h$ is of polynomial growth, $S_{1}$ and $S_{2}$ are positive stable and compatible in (A.3).

Proof. (A.1): Using the integration by parts entrywise and putting them in matrix form

$$
\begin{aligned}
& \mathcal{E}_{S_{1}}\left\{S_{1} h+h^{\prime}\right\}=S_{1} e^{-S_{1} x} \int_{0}^{x} e^{S_{1} y} h(y) \mathrm{d} y+e^{-S_{1} x} \int_{0}^{x} e^{S_{1} y} \mathrm{~d} h(y) \\
= & S_{1} e^{-S_{1} x} \int_{0}^{x} e^{S_{1} y} h(y) \mathrm{d} y+h-e^{-S_{1} x} h(0)-e^{-S_{1} x} \int_{0}^{x} \mathrm{~d} e^{S_{1} x} h(y)=h-e^{-S_{1} x} h(0) .
\end{aligned}
$$

(A.2): Changing the order of integration, we have

$$
\begin{aligned}
\mathcal{E}_{S_{1}}\left(S_{1}-S_{2}\right) \mathcal{E}_{S_{2}} h & =\int_{0}^{x} e^{-S_{1}(x-y)}\left(S_{1}-S_{2}\right) \int_{0}^{y} e^{-S_{2}(y-z)} h(z) \mathrm{d} z \mathrm{~d} y \\
& =\int_{0}^{x} e^{-S_{1} x}\left(\int_{z}^{x} e^{S_{1} y}\left(S_{1}-S_{2}\right) e^{-S_{2} y} \mathrm{~d} y\right) e^{S_{2} z} h(z) \mathrm{d} z .
\end{aligned}
$$

Note that

$$
\frac{\mathrm{d}}{\mathrm{d} y} e^{S_{1} y} e^{-S_{2} y}=e^{S_{1} y}\left(S_{1}-S_{2}\right) e^{-S_{2} y},
$$

which implies that

$$
\int_{z}^{x} e^{S_{1} y}\left(S_{1}-S_{2}\right) e^{-S_{2} y} \mathrm{~d} y=e^{S_{1} x} e^{-S_{2} x}-e^{S_{1} z} e^{-S_{2} z}
$$

Therefore we get

$$
\mathcal{E}_{S_{1}}\left(S_{1}-S_{2}\right) \mathcal{E}_{S_{2}} h=\int_{0}^{x} e^{-S_{2}(x-z)} h(z) \mathrm{d} z-\int_{0}^{x} e^{-S_{1}(x-z)} h(z) \mathrm{d} z,
$$

which completes the proof.

(A.3): Substituting $w=u+v$, we have

$$
\begin{aligned}
\mathcal{T}_{S_{1}}\left(S_{2}-S_{1}\right) \mathcal{T}_{S_{2}} h & =\int_{0}^{\infty} \int_{0}^{\infty} e^{-u S_{1}}\left(S_{2}-S_{1}\right) e^{-v S_{2}} h(x+u+v) \mathrm{d} u \mathrm{~d} v \\
& =\int_{0}^{\infty}\left(\int_{0}^{w} e^{-u S_{1}}\left(S_{2}-S_{1}\right) e^{u S_{2}} \mathrm{~d} u\right) e^{-w S_{2}} h(x+w) \mathrm{d} w .
\end{aligned}
$$


Note that

$$
\frac{\mathrm{d}}{\mathrm{d} u} e^{-u S_{1}} e^{u S_{2}}=e^{-u S_{1}}\left(S_{2}-S_{1}\right) e^{u S_{2}}
$$

which implies

$$
\int_{0}^{w} e^{-u S_{1}}\left(S_{2}-S_{1}\right) e^{u S_{2}} \mathrm{~d} u=e^{-w S_{1}} e^{w S_{2}}-I .
$$

Therefore, we get

$$
\mathcal{T}_{S_{1}}\left(S_{2}-S_{1}\right) \mathcal{T}_{S_{2}} h=\int_{0}^{\infty} e^{-w S_{1}} h(x+w) \mathrm{d} w-\int_{0}^{\infty} e^{-w S_{2}} h(x+w) \mathrm{d} w
$$

completing the proof.

Lemma A.2. For any positive stable matrices $S_{1}, S_{2}$ compatible with a matrix $Q$, and any vector function $h$ of polynomial growth, the following identities hold true.

$$
\begin{aligned}
\mathcal{T}_{S_{1}}\left(S_{1}-\mathcal{D}\right) h & =h ; \\
\mathcal{T}_{S_{1}}\left(S_{1} Q+Q S_{2}\right) \mathcal{E}_{S_{2}} h & =\mathcal{T}_{S_{1}} Q h+Q \mathcal{E}_{S_{2}} h, \\
\mathcal{E}_{S_{1}}\left(S_{1} Q+Q S_{2}\right) \mathcal{T}_{S_{2}} h & =\mathcal{E}_{S_{1}} Q h+Q \mathcal{T}_{S_{2}} h-e^{-S_{2} \cdot} Q \mathcal{L} h\left(S_{2}\right) .
\end{aligned}
$$

Proof. We first note that, for $j=1,2$,

$$
\lim _{y \rightarrow 0} e^{-S_{j} y} h(y)=0
$$

(A.4): By the integration-by-parts, it follows from (A.7) that

$$
\begin{aligned}
\mathcal{T}_{S_{1}}\left(S_{1}-\mathcal{D}\right) h & =e^{S_{1} x} \int_{x}^{\infty} e^{-S_{1} y} S_{1} h(y) \mathrm{d} y-e^{S_{1} x} \int_{x}^{\infty} e^{-S_{1} y} \mathrm{~d} h(y) \\
& =e^{S_{1} x} \int_{x}^{\infty} e^{-S_{1} y} S_{1} h(y) \mathrm{d} y-\left\{-h(x)+e^{S_{1} x} \int_{x}^{\infty} e^{-S_{1} y} S_{1} h(y) \mathrm{d} y\right\}=h .
\end{aligned}
$$

(A.5): We prove similarly by changing the order of integrations

$$
\begin{aligned}
\mathcal{T}_{S_{1}}\left(S_{1} Q+Q S_{2}\right) \mathcal{E}_{S_{2}} h= & \int_{0}^{\infty} e^{-S_{1} y}\left(S_{1} Q+Q S_{2}\right) \int_{0}^{x+y} e^{-S_{2}(x+y-z)} h(z) \mathrm{d} z \mathrm{~d} y \\
= & \int_{0}^{x}\left(\int_{0}^{\infty} e^{-S_{1} y}\left(S_{1} Q+Q S_{2}\right) e^{-S_{2} y} \mathrm{~d} y\right) e^{-S_{2}(x-z)} h(z) \mathrm{d} z \\
& +\int_{x}^{\infty}\left(\int_{z-x}^{\infty} e^{-S_{1} y}\left(S_{1} Q+Q S_{2}\right) e^{-S_{2} y} \mathrm{~d} y\right) e^{-S_{2}(x-z)} h(z) \mathrm{d} z .
\end{aligned}
$$

Because of (A.7), we must have

$$
\int_{x}^{\infty} e^{-S_{1} y}\left(S_{1} Q+Q S_{2}\right) e^{-S_{2} y} \mathrm{~d} y=e^{-S_{1} x} Q e^{-S_{2} x}
$$

Therefore,

$$
\mathcal{T}_{S_{1}}\left(S_{1}+S_{2}\right) \mathcal{E}_{S_{2}} h=Q \int_{0}^{x} e^{-S_{2}(x-z)} h(z) \mathrm{d} z+\int_{x}^{\infty} e^{-S_{1}(z-x)} Q h(z) \mathrm{d} z .
$$


(A.6): We prove in a similar manner that

$$
\begin{aligned}
& \mathcal{E}_{S_{1}}\left(S_{1} Q+Q S_{2}\right) \mathcal{T}_{S_{2}} h \\
= & e^{-S_{1} x} \int_{0}^{x}\left(\int_{0}^{z} e^{S_{1} y}\left(S_{1} Q+Q S_{2}\right) e^{S_{2} y}\right) e^{-S_{2} z} h(z) \mathrm{d} z \\
& +e^{-S_{1} x} \int_{x}^{\infty}\left(\int_{0}^{x} e^{S_{1} y}\left(S_{1} Q+Q S_{2}\right) e^{S_{2} y}\right) e^{-S_{2} z} h(z) \mathrm{d} z \\
= & e^{-S_{1} x} \int_{0}^{x}\left(e^{S_{1} z} Q-Q e^{-S_{2} z}\right) h(z) \mathrm{d} z+\int_{x}^{\infty}\left(Q e^{S_{2} x}-e^{-S_{1} x} Q\right) e^{-S_{2} z} h(z) \mathrm{d} z,
\end{aligned}
$$

which yields the identity after rearrangement.

Lemma A.3. Suppose that $S$ is diagonalizable and positive stable. Then it holds that

$$
\begin{aligned}
\mathcal{L}\left\{\mathcal{T}_{S} H_{\nu}\right\}(r)=(r I-S)^{-1}\left[\psi_{S+L}(S)+\mathcal{L}\{D \circ F\}(S)-\psi_{S+L}(r I)-\right. & \mathcal{L}\{D \circ F\}(r I)] \cdot \mathcal{L} H(r) \\
& +\mathcal{L}\left[\mathcal{T}_{S}\{N(S) H\}\right](r),
\end{aligned}
$$

where the constant matrix $N(S)=\psi_{S+L}(S)+\mathcal{L}\{D \circ F\}(S)$. Furthermore, if $\Delta_{\nu^{z}} \equiv 0$, then

$$
\mathcal{T}_{S}\left\{H_{\nu}\right\}=\mathcal{T}_{S}\{N(S) H\}+\left[\mathcal{T}_{S} \Delta_{\nu}+\mathcal{T}_{S}\{D \circ F\}\right] * H .
$$

Proof. Since $S$ is diagonalizable, there must exists a matrix $\Phi$ such that $S=\Phi^{-1} \operatorname{diag}\left(s_{1}, \cdots, s_{m}\right) \Phi$ where $s_{1}, \cdots, s_{m}>0$. We denote the three terms on the right-hand side of (3.5) by $H_{1}, H_{2}$ and $\mathrm{H}_{3}$ respectively. Firstly,

$$
\begin{aligned}
\mathcal{T}_{S} H_{1}(u)= & e^{S u} \int_{u}^{\infty} e^{-S y}\left[\int_{0}^{\infty} \Delta_{\nu^{S}}(\mathrm{~d} z)\{H(y-z)-H(y)\}\right] \mathrm{d} y \\
= & e^{S u} \int_{u}^{\infty} e^{-S y}\left[\int_{0}^{\infty}\left\{\Delta_{\nu^{S}}(\mathrm{~d} z) H(y-z)-\left(I-e^{-S z}+e^{-S z}\right) \Delta_{\nu^{S}}(\mathrm{~d} z) H(y)\right\}\right] \mathrm{d} y \\
= & e^{S u} \int_{u}^{\infty} e^{-S y}\left[\int_{0}^{\infty}\left\{\Delta_{\nu^{S}}(\mathrm{~d} z) H(y-z)-e^{-S z} \Delta_{\nu^{S}}(\mathrm{~d} z) H(y)\right\}\right] \mathrm{d} y \\
& +\mathcal{T}_{S}\left\{\psi_{S}(S) H\right\}(u) .
\end{aligned}
$$

Hence we need to evaluate the first term which we shall denote by $J_{1}(u)$.

$$
\begin{aligned}
J_{1}(u) & =e^{S u} \int_{0}^{\infty}\left[\int_{u}^{\infty} \mathrm{d} y e^{-S y} \Delta_{\nu^{S}}(\mathrm{~d} z) H(y-z)-\int_{u}^{\infty} \mathrm{d} y e^{-S(y+z)} \Delta_{\nu^{S}}(\mathrm{~d} z) H(y)\right] \\
& =e^{S u} \int_{0}^{\infty}\left[\int_{u}^{\infty} \mathrm{d} y e^{-S y} \Delta_{\nu^{S}}(\mathrm{~d} z) H(y-z)-\int_{u+z}^{\infty} \mathrm{d} y e^{-S y} \Delta_{\nu^{S}}(\mathrm{~d} z) H(y-z)\right] \\
& =e^{S u} \int_{0}^{\infty}\left[\int_{u}^{u+z} \mathrm{~d} y e^{-S y} \Delta_{\nu^{S}}(\mathrm{~d} z) H(y-z)\right] \\
& =e^{S u} \int_{0}^{\infty}\left[\int_{u-z}^{u} \mathrm{~d} x e^{-S(x+z)} \Delta_{\nu^{S}}(\mathrm{~d} z) H(x)\right] \\
& =\int_{0}^{u} \mathrm{~d} x e^{S(u-x)} \int_{u-x}^{\infty} e^{-S z} \Delta_{\nu^{S}}(\mathrm{~d} z) H(x) \\
& =\int_{0}^{u} \mathrm{~d} y e^{S y} \int_{y}^{\infty} e^{-S z} \Delta_{\nu^{S}}(\mathrm{~d} z) H(u-y)=\mathcal{T}_{\Delta_{\nu}} * H(u),
\end{aligned}
$$

where we have used many times the fact that $H(x)=0$ when $x<0$. To summarize,

$$
\mathcal{T}_{S} H_{1}(u)=\mathcal{T}_{\Delta_{\nu}} * H(u)+\mathcal{T}_{S}\left\{\psi_{S}(S) H\right\}(u),
$$


or equivalently,

$$
\mathcal{L}\left\{\mathcal{T}_{S} H_{1}\right\}(r)=(r I-S)^{-1}\left[\psi_{S}(S)-\psi_{S}(r I)\right] \cdot \mathcal{L} H(r)+\mathcal{L}\left\{\mathcal{T}_{S}\left\{\psi_{S}(S) H\right\}\right\}(r) .
$$

Secondly,

$$
\begin{aligned}
& \mathcal{T}_{S} H_{2}(u)=e^{S u} \int_{u}^{\infty} e^{-S y}\left[\int_{0}^{\infty} \Delta_{\nu^{Z}}(\mathrm{~d} z)\left\{H(y-z)-H(y)+z H^{\prime}(y)\right\}\right] \mathrm{d} y \\
= & e^{S u} \int_{u}^{\infty} e^{-S y}\left[\int _ { 0 } ^ { \infty } \Delta _ { \nu ^ { Z } } ( \mathrm { d } z ) \left\{H(y-z)-\left(I-e^{-S z}-S z+e^{-S z}+S z\right) \Delta_{\nu^{Z}}(\mathrm{~d} z) H(y)\right.\right. \\
& \left.+z \Delta_{\nu^{Z}}(\mathrm{~d} z) H^{\prime}(y)\right] \mathrm{d} y \\
= & e^{S u} \int_{u}^{\infty} e^{-S y}\left[\int _ { 0 } ^ { \infty } \Delta _ { \nu ^ { Z } } ( \mathrm { d } z ) \left\{H(y-z)-\left(e^{-S z}+S z\right) \Delta_{\nu^{Z}}(\mathrm{~d} z) H(y)\right.\right. \\
& \left.+z \Delta_{\nu^{Z}}(\mathrm{~d} z) H^{\prime}(y)\right] \mathrm{d} y+\mathcal{T}_{S}\left\{\psi_{L}(S) H\right\}(u) .
\end{aligned}
$$

We simplify the first term denoted by $J_{2}(u)$.

$$
\begin{aligned}
& J_{2}(u)=e^{S u} \int_{0}^{\infty}\left[\int_{u}^{\infty} e^{-S y} \Delta_{\nu^{Z}}(\mathrm{~d} z) H(y-z) \mathrm{d} y-\int_{u}^{\infty} e^{-S y}\left(e^{-S z}+S z\right) \Delta_{\nu^{Z}}(\mathrm{~d} z) H(y) \mathrm{d} y\right. \\
& \left.+\int_{u}^{\infty} e^{-S y} z \Delta_{\nu^{Z}}(\mathrm{~d} z) H^{\prime}(y) \mathrm{d} y\right] \\
= & e^{S u} \int_{0}^{\infty}\left[\int_{u}^{\infty} e^{-S y} \Delta_{\nu^{Z}}(\mathrm{~d} z) H(y-z) \mathrm{d} y-\int_{u}^{\infty} e^{-S(y+z)} \Delta_{\nu^{Z}}(\mathrm{~d} z) H(y) \mathrm{d} y-e^{-S u} z \Delta_{\nu^{Z}}(\mathrm{~d} z) H(u)\right] \\
= & e^{S u} \int_{0}^{\infty}\left[\int_{u}^{u+z} e^{-S y} \Delta_{\nu^{z}}(\mathrm{~d} z) H(y-z) \mathrm{d} y-e^{-S u} z \Delta_{\nu^{z}}(\mathrm{~d} z) H(u)\right] .
\end{aligned}
$$

In the penultimate equality we use the fact that $\lim _{y \rightarrow \infty} e^{-S y} \Delta_{\nu}(\mathrm{d} z) H(y)=0$, which follows from (A.7) and that $H$ is bounded and $\nu_{i}^{Z}$ is finite on a compact set apart from the origin for all $i \in E$. Applying Laplace transforms yields

$$
\begin{aligned}
& \mathcal{L}\left\{J_{2}\right\}(r)=\int_{0}^{\infty}\left[\int_{0}^{\infty} e^{-(r I+S) u} \int_{u-z}^{u} e^{-S(w+z)} \Delta_{\nu^{Z}}(\mathrm{~d} z) H(w) \mathrm{d} w \mathrm{~d} u-\int_{0}^{\infty} e^{-r u} z \Delta_{\nu^{Z}}(\mathrm{~d} z) H(u)\right] \\
= & \int_{0}^{\infty}\left[(r I-S)^{-1}\left[e^{-(r I-S) w}-e^{-(r I-S)(w+z)}\right] e^{-S(w+z)} \Delta_{\nu^{Z}}(\mathrm{~d} z) H(w) \mathrm{d} w-z \Delta_{\nu^{Z}}(\mathrm{~d} z) \mathcal{L} H(r)\right] \\
= & \int_{0}^{\infty}\left[(r I-S)^{-1}\left[e^{-(r w-S) z}-e^{-r(w+z)}\right] \Delta_{\nu^{Z}}(\mathrm{~d} z) H(w) \mathrm{d} w-z \Delta_{\nu^{Z}}(\mathrm{~d} z) \mathcal{L} H(r)\right] \\
= & \int_{0}^{\infty}\left[(r I-S)^{-1}\left[e^{-S z} \Delta_{\nu^{Z}}(\mathrm{~d} z) \mathcal{L} H(r)(r)-e^{-r z} \Delta_{\nu^{Z}}(\mathrm{~d} z) \mathcal{L} H(r)(r)\right]-z \Delta_{\nu^{Z}}(\mathrm{~d} z) \mathcal{L} H(r)\right] \\
= & (r I-S)^{-1} \int_{0}^{\infty}\left[e^{-S z}+S z-I+e^{-r z} I+r z I-I\right] \Delta_{\nu^{Z}}(\mathrm{~d} z) \mathcal{L} H(r) \\
= & (r I-S)^{-1}\left[\psi_{L}(S)-\psi_{L}(r I)\right] \cdot \mathcal{L} H(r) .
\end{aligned}
$$

Thus,

$$
\mathcal{L}\left\{\mathcal{T}_{S} h_{2}\right\}(r)=(r I-S)^{-1}\left[\psi_{L}(S)-\psi_{L}(r I)\right] \cdot \mathcal{L} H(r)+\mathcal{L}\left\{\mathcal{T}_{S}\left\{\psi_{L}(S) H\right\}\right\}(r) .
$$

Finally, we see that

$$
\begin{aligned}
& \mathcal{T}_{S} H_{3}(u)=e^{S u} \int_{u}^{\infty} e^{-S y} \int_{0}^{y} D \circ F(y-w) H(w) \mathrm{d} w \mathrm{~d} y \\
= & e^{S u} \int_{0}^{u} \int_{u-w}^{\infty} e^{-S(y+w)} D \circ F(y) \mathrm{d} y H(w) \mathrm{d} w+e^{S u} \int_{u}^{\infty} \int_{w}^{\infty} e^{-S y} D \circ F(y-w) \mathrm{d} y H(w) \mathrm{d} w \\
= & \mathcal{T}_{S}\{D \circ F\} * H(u)+\mathcal{T}_{S}\{\mathcal{L}\{D \circ F\}(S) H\}(u) .
\end{aligned}
$$


Thus,

$\mathcal{L}\left\{\mathcal{T}_{S} H_{3}\right\}(r)=(r I-S)^{-1}[\mathcal{L}\{D \circ F(S)\}-\mathcal{L}\{D \circ F(r I)\}] \cdot \mathcal{L} H(r)+\mathcal{L}\left[\mathcal{T}_{S}\{\mathcal{L}\{D \circ F\}(S) H\}\right](r)$

Combining (A.10), (A.11) and (A.12) gives the identity (A.8). When $\Delta_{\nu^{z}} \equiv 0$, then $J_{2} \equiv 0$ and hence the identity (A.9) follows immediately.

\section{B Detailed proofs}

Proof of Lemma 2.2: Let $F^{\delta}(s):=K(s I)-\delta I$. The assumption means that the multiplicity of $\rho_{j}$ is 1 , which implies that a Jordan pair $\left(v_{j}, \rho_{j}\right)$ in the sense of Definition 2 in D'Auria et al. [16] satisfies $F^{\delta}\left(\rho_{j}\right) v_{j}=0$, that is, each $v_{j}$ is the first Jordan chain corresponding to $\rho_{j}$. Then, by Theorem 1 in D'Auria et al. [16], we find that

$$
v=\left[v_{1}, \ldots, v_{m}\right], \quad \Gamma=\operatorname{diag}\left[\rho_{1}, \ldots, \rho_{m}\right]
$$

are $m \times m$ invertible matrices. Using these, we shall construct

$$
R:=\left(v^{\top}\right)^{-1} \Gamma v^{\top}
$$

Then

$$
\begin{aligned}
v^{\top}[K(R)-\delta I]= & v^{\top} C+\Gamma^{2} v^{\top} \Delta_{\sigma^{2} / 2}+\Gamma v^{\top} \Delta_{\mu}+\int_{0}^{\infty}\left(e^{-z \Gamma}-I\right) v^{\top} \Delta_{\nu^{S}}(\mathrm{~d} z) \\
& +\int_{0}^{\infty}\left(e^{-z \Gamma}-I+z \Gamma\right) v^{\top} \Delta_{\nu^{z}}(\mathrm{~d} z)+\int_{0}^{\infty} e^{-z \Gamma} v^{\top} D \circ F(z) \mathrm{d} z-\delta v^{\top} .
\end{aligned}
$$

Observing the rows of the above matrix, we see that, for each $i=1, \ldots, m$,

$$
\begin{aligned}
& v_{i}^{\top} C+\rho_{i}^{2} v_{i}^{\top} \Delta_{\sigma^{2} / 2}+\rho_{i} v_{i}^{\top} \Delta_{\mu}+\int_{0}^{\infty}\left(e^{-z \rho_{i}}-1\right) v_{i}^{\top} \Delta_{\nu}(\mathrm{d} z) \\
& \quad+\int_{0}^{\infty}\left(e^{-z \rho_{i}}-1+z \rho_{i}\right) v_{i}^{\top} \Delta_{\nu^{Z}}(\mathrm{~d} z)+\int_{0}^{\infty} e^{-z \rho_{i}} v_{i}^{\top} D \circ F(z) \mathrm{d} z-\delta v^{\top} \\
& =v_{i}^{\top}\left[K\left(\rho_{i}\right)-\delta I\right]=(0, \ldots, 0),
\end{aligned}
$$

which implies that $v^{\top}[K(R)-\delta I]=0$. Since $v^{\top}$ is invertible, we conclude that $K(R)-\delta I=0$. Note that $R$ is diagonalizable by definition, and is positive stable since $\operatorname{Re}\left(\rho_{j}\right)>0$.

Proof of Lemma 3.1: Let $\sigma:=\inf \left\{t: J_{t} \neq i, J_{0}=i\right\}$ and $S_{n}=\inf \left\{t: X_{t} \notin(x-1 / n, x+1 / n)\right\}$ for sufficiently large $n$ such that $x-1 / n>0$. Using the usual arguments involving the strong Markov property (similar to the proof of Theorem 2.1 of Feng [21]), we can show that

$$
\mathbb{E}^{(x, i)}\left[e^{-\delta\left(\sigma \wedge S_{n}\right)} H\left(X_{\sigma \wedge S_{n}}, J_{\sigma \wedge S_{n}}\right)\right]-H(x, i)=-\mathbb{E}^{(x, i)}\left[\int_{0}^{\sigma \wedge S_{n}} e^{-\delta s} l\left(X_{s}, J_{s}\right) \mathrm{d} s\right] .
$$

Denote the left-hand side of (B.2) by $h_{1}$ and the RHS by $-h_{2}$. Moreover, since $X_{t}=X_{t}^{(i)}$ for $t \in[0, \sigma)$, where $\left\{X_{t}^{(i)}, t \geq 0\right\}$ is a Lévy process with the characteristic triple $\left(\sigma_{i}, \mu_{i}, \nu_{i}^{S+Z}\right)$, one can show in a manner similar to Lemma A.1 of Feng [21] that

$$
\mathbb{E}^{(x, i)}\left[e^{-\delta\left(\sigma \wedge S_{n}\right)} H\left(X_{\left(\sigma \wedge S_{n}\right)_{-}}^{(i)}, i\right)\right]=H(x, i)+\mathbb{E}^{(x, i)}\left[\int_{0}^{\sigma \wedge S_{n}} e^{-\delta s}(\mathcal{A}-\delta) H\left(X_{s}^{(i)}, i\right) \mathrm{d} s\right],
$$


where $\mathcal{A}$ is the infinitesimal generator of $X^{(i)}$ given by

$$
\begin{aligned}
\mathcal{A} h(x, i)= & \frac{\sigma_{i}^{2}}{2} h^{\prime \prime}(x, i)+\mu_{i} h^{\prime}(x, i)+\int_{0}^{\infty}\{h(x-z, i)-h(x, i)\} \nu^{S}(\mathrm{~d} z) \\
& +\int_{0}^{\infty}\left\{h(x-z, i)-h(x, i)+z h^{\prime}(x, i)\right\} \nu_{i}^{Z}(\mathrm{~d} z) .
\end{aligned}
$$

Thus, we obtain that

$$
\begin{aligned}
h_{1}(x, i)= & \mathbb{E}^{(x, i)}\left[e^{-\delta\left(\sigma \wedge S_{n}\right)}\left(H\left(X_{\sigma \wedge S_{n}}, J_{\sigma \wedge S_{n}}\right)-H\left(X_{\left(\sigma \wedge S_{n}\right)-}^{(i)}, i\right)\right)\right] \\
& +\mathbb{E}^{(x, i)}\left[e^{-\delta\left(\sigma \wedge S_{n}\right)} H\left(X_{\left(\sigma \wedge S_{n}\right)-}^{(i)}, i\right)\right]-H(x, i) \\
= & \mathbb{E}^{(x, i)}\left[e^{-\delta\left(\sigma \wedge S_{n}\right)}\left(H\left(X_{\sigma \wedge S_{n}}, J_{\sigma \wedge S_{n}}\right)-H\left(X_{\left(\sigma \wedge S_{n}\right)-}^{(i)}, i\right)\right)\right] \\
& +\mathbb{E}^{(x, i)}\left[\int_{0}^{\sigma \wedge S_{n}} e^{-\delta s}(\mathcal{A}-\delta) H\left(X_{s}^{(i)}, i\right) \mathrm{d} s\right]
\end{aligned}
$$

Note that the first transition in $J$ from $i$ to $j$ can be viewed as the first event of a Poisson random measure $N_{i j}$ independent of $X$ with the intensity measure $\nu_{i j}$ for which $\nu_{i j}(\mathrm{~d} y)=$ $\lambda_{i j} q_{i j} f_{i j}(y) \mathrm{d} y+\lambda_{i j}\left(1-q_{i j}\right) \delta_{0}(y) \mathrm{d} y$ for $i, j \in E$, where $\delta_{0}$ is the Dirac function on $\{0\}$. Therefore, we have

$$
\begin{aligned}
& \mathbb{E}^{(x, i)}\left[e^{-\delta\left(\sigma \wedge S_{n}\right)}\left(H\left(X_{\sigma \wedge S_{n}}, J_{\sigma \wedge S_{n}}\right)-H\left(X_{\left(\sigma \wedge S_{n}\right)-}, i\right)\right)\right] \\
&=\sum_{j \in E} \mathbb{E}^{(x, i)}\left[\int_{0}^{\sigma \wedge S_{n}} e^{-\delta s} \int_{0}^{\infty}\left(H\left(X_{s-}^{(i)}-y, j\right)-H\left(X_{s-}^{(i)}, i\right)\right) N_{i j}(\mathrm{~d} s, \mathrm{~d} y)\right] \\
&+\sum_{j \in E} \mathbb{E}^{(x, i)}\left[\int_{0}^{\sigma \wedge S_{n}} e^{-\delta s}\left(H\left(X_{s}^{(i)}, j\right)-H\left(X_{s}^{(i)}, i\right)\right) \lambda_{i j}\left(1-q_{i j}\right) \mathrm{d} s\right] \\
&= \sum_{j \in E} \mathbb{E}^{(x, i)}\left[\int_{0}^{\sigma \wedge S_{n}} e^{-\delta s}\left(\int_{0}^{\infty} H\left(X_{s}^{(i)}-y, j\right) \lambda_{i j} q_{i j} f_{i j}(y) \mathrm{d} y+H\left(X_{s}^{(i)}, j\right) \lambda_{i j}\left(1-q_{i j}\right)\right) \mathrm{d} s\right] \\
&= \sum_{j \in E} D_{i j} \mathbb{E}^{(x, i)}\left[\int_{0}^{\sigma \wedge S_{n}} e^{-\delta s} f_{i j}\left(X_{s}^{(i)}-z\right) H(z, j) \mathrm{d} z \mathrm{~d} s\right]+\sum_{j \in E} C_{i j} \mathbb{E}^{(x, i)}\left[\int_{0}^{\sigma \wedge S_{n}} e^{-\delta s} H\left(X_{s}^{(i)}, j\right) \mathrm{d} s\right] .
\end{aligned}
$$

For notational convenience, we let

$$
\mathcal{B} h(x, i)=\mathcal{A} h(x, i)+\sum_{j \in E}\left(C_{i j} h(x, j)+D_{i j} \int_{0}^{\infty} f_{i j}(x-z) h(z, j) \mathrm{d} z\right)
$$

Combining (B.3) and (B.4), we obtain

$$
h_{1}(x, i)=\int_{0}^{\sigma \wedge S_{n}} e^{-\delta s}(\mathcal{B}-\delta) H\left(X_{s}^{(i)}, i\right) \mathrm{d} s
$$


Hence, on one hand,

$$
\begin{aligned}
\lim _{n \rightarrow \infty} \mid & \left|\frac{h_{1}(x, i)}{\left(1-\mathbb{E}^{x}\left[e^{-\delta\left(\sigma \wedge S_{n}\right)}\right]\right) / \delta}-(\mathcal{B}-\delta I) H(x, i)\right| \\
\quad & \lim _{n \rightarrow \infty}\left|\frac{\mathbb{E}^{(x, i)}\left[\int_{0}^{\sigma \wedge S_{n}} e^{-\delta s}\left\{(\mathcal{B}-\delta I) H\left(X_{s}^{(i)}, J_{s}\right)-\mathcal{B} H(x, i)+\delta H(x, i)\right\} \mathrm{d} s\right]}{\left(1-\mathbb{E}^{x}\left[e^{-\delta\left(\sigma \wedge S_{n}\right)}\right]\right) / \delta}\right| \\
& \leq \lim _{n \rightarrow \infty} \frac{\mathbb{E}^{(x, i)}\left[\int_{0}^{\sigma \wedge S_{n}} e^{-\delta s}\left|(\mathcal{B}-\delta I) H\left(X_{s}^{(i)}, i\right)-\mathcal{B} H(x, i)+\delta H(x, i)\right| \mathrm{d} s\right]}{\left(1-\mathbb{E}^{x}\left[e^{-\delta\left(\sigma \wedge S_{n}\right)}\right]\right) / \delta} \\
& \leq \lim _{n \rightarrow \infty} \sup _{y \in(x-1 / n, x+1 / n)}|\{\mathcal{B} H(y, i)-\delta H(y, i)\}-\mathcal{B} H(x, i)+\delta H(x, i)|=0,
\end{aligned}
$$

with the second to last inequality arising from Jensen's inequality and the last inequality from the fact that $(\mathcal{B}-\delta) H(x, i)$ is continuous in $x$ and $X_{s} \in(x-1 / n, x+1 / n)$ which is bounded by a compact set.

On the other hand,

$$
\lim _{n \rightarrow \infty}\left|\frac{h_{2}(x, i)}{\left(1-\mathbb{E}^{(x, i)}\left[e^{-\delta\left(S_{n} \wedge \sigma\right)}\right]\right) / \delta}-l(x, i)\right| \leq \lim _{n \rightarrow \infty} \sup _{y \in(x-1 / n, x+1 / n)}|l(y, i)-l(x, i)|=0,
$$

due to the continuity of $l(x, i)$ for $x \notin D$. Since $h_{1} \equiv-h_{2}$ by (B.2), we can conclude that

$$
(\mathcal{B}-\delta I) H(x, i)=-l(x, i), \quad x \notin D, i \in E
$$

which is the desired result (3.5).

\section{Proof of Corollary 3.1:}

When $\Delta_{\sigma^{2}}=0,(3.2)$ reduces to

$$
\left\{\Delta_{\mu} D-(\delta I-C)\right\} H(x)+\left\{H_{\nu}+l\right\}(x)=0, \quad x>0,
$$

or equivalently,

$$
\left\{(\delta I-C) \Delta_{\mu}^{-1}-D\right\} \Delta_{\mu} H=H_{\nu}+l .
$$

Note that the matrix $(\delta I-C) \Delta_{\mu}^{-1}$ is positive stable under (A2). Applying (A.4) gives

$$
\Delta_{\mu} H=\mathcal{T}_{S}\left(H_{\nu}+l\right), \quad S:=(\delta I-C) \Delta_{\mu}^{-1} .
$$

Applying (A.3) and using the identity (B.5) leads to

$$
\mathcal{T}_{S}\left(H_{\nu}+l\right)=\mathcal{T}_{R}\left(H_{\nu}+l\right)-\mathcal{T}_{R}(S-R) \mathcal{T}_{S}\left(H_{\nu}+l\right)=\mathcal{T}_{R}\left(H_{\nu}+l\right)-\mathcal{T}_{R}(S-R) \Delta_{\mu} H .
$$

We expand the first term on the RHS of (B.6) according to (A.9),

$$
\mathcal{T}_{R}\left(H_{\nu}+l\right)=\mathcal{T}_{R}\left\{\psi_{S+L}(R)+\mathcal{L}\{D \circ F\}(R)\right\} H+\left[\mathcal{T}_{R} \Delta_{\nu}+\mathcal{T}_{R}\{D \circ F\}\right] * H+\mathcal{T}_{R} l .
$$

Note that (2.3) implies

$$
(S-R) \Delta_{\mu}=\psi_{S+L}(R)+\mathcal{L}\{D \circ F\}(R) .
$$

Thus, the second term on the RHS of (B.6) is given by

$$
\mathcal{T}_{R}(S-R) \Delta_{\mu} H=\mathcal{T}_{R}\left\{\psi_{S+L}(R)+\mathcal{L}\{D \circ F\}(R)\right\} H .
$$

Therefore,

$$
\Delta_{\mu} H=\mathcal{T}_{S}\left(H_{\nu}+l\right)=\left[\mathcal{T}_{R} \Delta_{\nu}+\mathcal{T}_{R}\{D \circ F\}\right] * H+\mathcal{T}_{R} l,
$$

which yields (3.11) after pre-multiplying $\Delta_{\mu}^{-1}$. 


\section{Solution to a quadratic matrix equation}

Lemma C.1. Suppose that $(\delta I-C) \Delta_{\sigma^{2} / 2}^{-1}$ is non-singular. Then, for an $m \times m$ matrix $X, a$ quadratic matrix equation

$$
X^{2}-X \Delta_{2 \mu / \sigma^{2}}-(\delta I-C) \Delta_{\sigma^{2} / 2}^{-1}=0,
$$

has a positive stable solution.

Proof. Let $Y:=X^{\top}$, and consider the transposed equation of (C.1):

$$
Y^{2}-\Delta_{2 \mu / \sigma^{2}} Y-F^{\top}=0,
$$

where $F:=(\delta I-C) \Delta_{\sigma^{2} / 2}^{-1}$, which is non-singular. Thanks to Theorem 3.2 by Guo [25], if $F^{\top}$ is an $M$-matrix, then (C.2) has a solution $Y$ that is a non-singular M-matrix. Since it is true for any non-singular $M$-matrix that its transpose is also a non-singular $M$-matrix; see, e.g., Theorem 1.1 in Guo [25], if $F$ is an $M$-matrix then a solution $X=Y^{\top}$ is also a non-singular $M$-matrix, which implies that the eigenvalues of $X$ have positive real parts by Theorem 1.1 in Guo [25] again. Hence the proof is complete if we show that $F$ is an $M$-matrix, which is equivalent to stating that there exists some positive diagonal matrix $D$ such that $F D=\left(\gamma_{i j}\right)$ is strictly row diagonally dominant:

$$
\left|\gamma_{i i}\right|>\sum_{j=1 ; j \neq i}^{m}\left|\gamma_{i j}\right|, \quad i \in E
$$

see Horn and Johnson [26], p.114, Theorem 2.5.3.13. To show (C.3) for $F$, we take $D=\Delta_{\sigma^{2} / 2}$ so that $F D=\delta I-C=\left(\gamma_{i j}\right)$. Note that, for each $i \in E$,

$$
\left|\gamma_{i i}\right|=\delta-\lambda_{i i} ; \quad\left|\gamma_{i j}\right|=\lambda_{i j}\left(1-q_{i j}\right)(j \neq i) .
$$

Since $\sum_{j=1}^{m} \lambda_{i j}=0$ by the property of the intensity matrix $\Lambda$, it is clear that

$$
\left|\gamma_{i i}\right| \geq-\lambda_{i i}=\sum_{j=1 ; j \neq i}^{m} \lambda_{i j}>\sum_{j=1 ; j \neq i}^{m}\left|\gamma_{i j}\right|,
$$

that is, $F$ is an $M$-matrix. This completes the proof.

\section{Semi-Markov matrix}

It is known from Markov renewal theory (c.f. Chinlar [12, Theorem 3.13]) that the Markov renewal equation (3.11) has a unique solution if $\int_{0}^{x} g(y) \mathrm{d} y$ is a semi-Markov matrix, i.e.

$$
\left\{\int_{0}^{\infty} g(z) \mathrm{d} z\right\} 1 \leq 1
$$

Although the proof appears technical, the idea is rather straightforward. It is easy to show that the function $g$ is semi-Markov for a MAP without a Gaussian component. In the general case, we extend the result using the weak convergence arguments developed in Gerber and Landry [23].

In the setting of Theorem 3.1, without loss of generality it is assumed $Z_{i}(t)=\sigma_{i} B_{i}(t)$ with $\sigma_{i}^{2}>0$ for $i \in E$. We first replace the Gaussian components by processes of the form

$$
Z_{i}^{(k)}(t)=d_{i}^{(k)} t-\int_{0}^{t} \int_{0}^{\infty} z N_{i}^{(k)}(\mathrm{d} s, \mathrm{~d} z), \quad k>0,
$$


where $N_{i}^{(k)}$ is a Poisson random measure with $\mathbb{E}\left[N_{i}^{(k)}(A, t)\right]=\lambda_{i}^{(k)} \Delta_{k}(A)$ for any Borel set $A$ on $(0, \infty)$ and $\Delta_{a}$ is the Dirac delta measure that assign measure one to the point $a$. In other words, $Z_{i}^{(k)}$ is a Poisson process with intensity $\lambda_{k}$ and fixed jump size of $k$. We set

$$
d_{i}^{(k)}=\frac{\sigma_{i}^{2}}{k}, \quad \lambda_{i}^{(k)}=\frac{\sigma_{i}^{2}}{k^{2}}, \quad i \in E .
$$

Then we see that, as $k \rightarrow 0$, the law of the process $\left\{\Sigma^{(k)}:=\left(Z_{i}^{(k)}\right)_{i \in E}\right\}$ converges weakly to the law of a Brownian motion $\left\{B:=\left(\sigma_{i} B_{i}\right)_{i \in E}\right\}$ in $\mathbb{D}(0, \infty)$ endowed with the Skorokhod topology; see, e.g., Jacod and Shiryayev [31], Corollary VII.3.6.

Consider the process $X_{i}^{(k)}(t)=u+\mu_{i} t-S^{(i)}(t)-Z_{i}^{(k)}(t)$ with $Z_{i}^{(k)}$ defined in (D.2), and denote the characteristic exponent matrix of $X^{(k)}$ by

$$
K^{(k)}(s)=C+\left\{s \Delta_{d^{(k)}}+\left(e^{-k s}-1\right) \Delta_{\lambda^{(k)}}\right\}+s \Delta_{\mu}+\psi_{S}(s)+\mathcal{L}\{D \circ F\}(s) .
$$

Our first aim is to find a positive stable matrix $R^{(k)}$ satisfying $K^{(k)}\left(R^{(k)}\right)=\delta I$, which converges to $R$ given in the proof of Lemma 2.2 .

Lemma D.1. For any $\rho_{j}(j \in E)$ given in Lemma 2.2, there exists some $\rho_{j}^{(k)}$ that is a solution to $\operatorname{det}\left[K^{(k)}(s I)-\delta I\right]=0$ such that $\rho_{j}^{(k)} \rightarrow \rho_{j}$, as $k \rightarrow 0$.

Proof. Due to the fact that $X^{(k)} \rightarrow^{d} X$ in $\mathbb{D}(0, \infty)$ and the choice of $d_{i}^{(k)}$ and $\lambda_{i}^{(k)}$, it follows that for any bounded subset $\mathcal{S} \subset\{z \in \mathbb{C} \mid \operatorname{Re}(z)>0\}$ and each $i, j \in E$ that

$$
\sup _{s \in \overline{\mathcal{S}}}\left|K_{i j}^{(k)}(s)-K_{i j}(s)\right| \rightarrow 0, \quad k \rightarrow 0
$$

where $K(s)$ is given in Lemma 2.2 with $\sigma_{i}^{2}>0$. Now considering analytic functions on $\mathbb{C}$

$$
\zeta^{(k)}(s):=\operatorname{det}\left[K^{(k)}(s I)-\delta I\right], \quad \zeta(s):=\operatorname{det}[K(s I)-\delta I] .
$$

We see from the above fact that $\zeta^{(k)}(s) \rightarrow \zeta(s)$ as $k \rightarrow 0$ uniformly in $s \in \overline{\mathcal{S}}$, and that the equation $\zeta^{(k)}(s)=0$ has $m$ roots, say $\left\{\rho_{i}^{(k)}, i=1, \ldots, m\right\}$ by the same argument as in the proof of Lemma 2.2. Hence, by Hurwitaz' theorem, we can choose a sequence $\rho_{i}^{(k)}$ in a neighborhood of $\rho_{j}$ such that $\rho_{i}^{(k)} \rightarrow \rho_{j}$ as $k \rightarrow 0$.

Lemma D.2. For each $k>0$, there exists a positive stable matrix $R^{(k)}$ satisfying $K^{(k)}\left(R^{(k)}\right)=$ $\delta I$. Let $R$ be the matrix given in Lemma 2.2. Then

$$
\lim _{k \rightarrow 0} R^{(k)}=R
$$

Proof. Using $\rho_{j}$ and $\rho_{j}^{(k)}$ given in Lemmas 2.2 and D.1, respectively, we denote by

$$
Q_{j}^{k}:=\left[K\left(\rho_{j}^{(k)} I\right)-\delta I\right]^{\top} ; \quad Q_{j}:=\left[K\left(\rho_{j} I\right)-\delta I\right]^{\top} .
$$

Then it follows that $Q_{j}^{k} \rightarrow Q_{j}(k \rightarrow 0)$ entrywise. Since $\operatorname{rank} Q_{j}^{k}<m$ for all $k>0$ from the definition of $\rho_{j}^{k}$, we can take $m$ non-zero vectors

$$
v_{j}^{(k)} \in \operatorname{Ker} Q_{j}^{k}, \quad j=1, \ldots, m
$$


Note that this $v_{j}^{(k)}$ can be taken so that as $k \rightarrow 0, v_{j}^{(k)} \not \nrightarrow 0$, by taking normalized sequence $\left|v_{j}^{(k)}\right|=1$. Thus, the entrywise limit of $v_{j}^{(k)}$ must belong to $\operatorname{Ker} Q_{j}$ because entrywise

$$
Q_{j}^{k} v_{j}^{(k)} \equiv 0, \quad Q_{j}^{k} \rightarrow Q_{j}
$$

Since $\rho_{j}$ 's are distinct, it follows that

$$
\operatorname{dim}\left(\operatorname{Ker} Q_{j}\right)=1, \quad j=1, \ldots, m .
$$

Then, we can take a sequence such that $v_{j}^{(k)} \rightarrow c_{j} v_{j}(k \rightarrow 0)$ entrywise for some $c_{j} \neq 0$, where $v_{j}$ is given in Lemma 2.2: $\left[K\left(\rho_{j} I\right)-\delta I\right]^{\top} v_{j}=0$. Using the sequence $v_{j}^{(k)}$, we can define matrices $v^{(k)}=\left(c_{1}^{-1} v_{1}^{(k)}, \ldots, c_{m}^{-1} v_{m}^{(k)}\right)$, which satisfies that $v^{(k)} \rightarrow v$ entrywise, where $v=\left(v_{1}, \ldots, v_{m}\right)$ is given in Lemma 2.2. It follows that

$$
\left|\operatorname{det} v^{(k)}\right| \rightarrow|\operatorname{det} v|>0, \quad k \rightarrow 0 .
$$

Since $\operatorname{det} v^{(k)}$ is continuous with respect to all components, there exists some $k_{0}>0$ such that $\left|\operatorname{det} v^{(k)}\right|>0$ for any $k<k_{0}$, which means that $v^{(k)}$ is non-singular for $k<k_{0}$. We can define $R^{(k)}:=\left(\left(v^{(k)}\right)^{\top}\right)^{-1} \Delta_{\rho^{(k)}}\left(v^{(k)}\right)^{\top}, 0<k<k_{0}$, where $\rho^{(k)}=\left(\rho_{1}^{(k)}, \ldots, \rho_{m}^{(k)}\right)^{\top}$. Thus, (D.3) is proved, and by the same argument as in the last half of the proof of Lemma 2.2, we also see that $K^{(k)}\left(R^{(k)}\right)=\delta I$.

Let $T:=\inf \left\{t>0 \mid X_{t}^{(k)}<0\right\}$, and let $F_{S}^{(k)}$ denote the vector of "discounted" survival functions of the deficit caused by jumps of the subordinator.

$$
F_{S}^{(k)}(x, z):=\mathbb{E}^{x}\left[e^{-\delta T} \varpi_{J_{T}, J_{T-}}\left(X_{T-}^{(k)}, X_{T}^{(k)}\right) I\left(\Delta X_{T}^{(k)}=S_{J_{T}}(T)>0, J_{T-}=J_{T}\right)\right],
$$

where

$$
\varpi_{(i, j)}(x, y)=I(y<-z), \quad z>0, \text { for } i, j \in E .
$$

Then it follows from Remark 5.1(2) and (3.11) that

$$
F_{S}^{(k)}(x, z)=\int_{0}^{x} g^{*}(y) F_{S}^{(k)}(x-y, z) \mathrm{d} y+\Delta_{\mu+d^{(k)}}^{-1} \mathcal{T}_{R^{(k)}} l,
$$

where $g^{*}$ is some matrix that is not needed in the following derivation,

$$
l(x)=\int_{0}^{\infty} \Delta_{\varpi}(x, x-y) \Delta_{\nu^{S}}(\mathrm{~d} y)=\int_{x+z}^{\infty} \Delta_{\nu^{S}}(\mathrm{~d} y)=\Delta_{\nu^{S}}(x+z, \infty) .
$$

Let $x=0$ in (D.4). Thus we note that

$$
F_{S}^{(k)}(0, z)=\Delta_{\mu+d^{(k)}}^{-1} \int_{0}^{\infty} e^{-R^{(k)} y} \Delta_{\nu^{S+Z^{(k)}}(y+z, \infty)} \mathrm{d} y .
$$

$F_{S}^{(k)}(0, z)$ can be interpreted as the "discounted" survival distribution of first record low caused by the subordinator. Therefore, its corresponding "discounted" density is given by

$$
F_{S}^{(k)}(z):=-\frac{\mathrm{d}}{\mathrm{d} z} F_{S}^{(k)}(0, z)=\Delta_{\mu+d^{(k)}}^{-1} \mathcal{T}_{R^{(k)}} \Delta_{\nu^{\prime}}(z),
$$

Similarly, we can define the vector of "discounted" survival functions of the deficit caused by jumps of fixed size $k$.

$$
F_{Z}^{(k)}(x, z):=\mathbb{E}^{x}\left[e^{-\delta T} \varpi_{J_{T}, J_{T-}}\left(X_{T-}^{(k)}, X_{T}^{(k)}\right) I\left(\Delta X_{T}^{(k)}=Z_{J_{T}}^{(k)}(T)=k, J_{T-}=J_{T}\right)\right] .
$$


Clearly, the "discounted" density of the first record low caused by a jump of fixed size $k$ is only defined on $(0, k]$ such that

$$
F_{Z}^{(k)}(z):=-\frac{\mathrm{d}}{\mathrm{d} z} F_{Z}^{(k)}(0, z)=\Delta_{\mu+d^{(k)}}^{-1} e^{-R^{(k)}(k-z)} I(0<z \leq k) \Delta_{\lambda^{(k)}} .
$$

We can also define the vector of "discounted" survival functions of the deficit caused by jumps associated with transitions of $J$,

$$
F_{J}^{(k)}(x, z):=\mathbb{E}^{x}\left[e^{-\delta T} \varpi_{J_{T}, J_{T-}}\left(X_{T-}^{(k)}, X_{T}^{(k)}\right) I\left(\Delta X_{T}^{(k)}>0, J_{T-} \neq J_{T}\right)\right] .
$$

Likewise we obtain the "discounted" density of the first record low due to transition,

$$
F_{J}^{(k)}(z):=-\frac{\mathrm{d}}{\mathrm{d} z} F_{J}^{(k)}(0, z)=\Delta_{\mu+d^{(k)}}^{-1} \mathcal{T}_{R^{(k)}}(D \circ F)(z) .
$$

Define

$$
g^{(k)}(z):=\sum_{n=0}^{\infty}\left(F_{Z}^{(k)}\right)^{* n} *\left\{F_{S}^{(k)}+F_{J}^{(k)}\right\}(z) .
$$

Thus, $g^{(k)}$ gives the "discounted" density of first record low due to either subordinator or Markov chain transition after countably many jumps of fixed size. Therefore, it must be true that $\int_{0}^{\infty} g^{(k)}(z) \mathrm{d} z \mathbf{1} \leq 1$. Then we need to show $g^{(k)}$ converges to $g$ in the general case where $\boldsymbol{\Sigma}^{(k)}$ is replaced by $B$. Recall that in the general case the matrix $R$ is determined by

$$
C+R^{2} \Delta_{\sigma^{2} / 2}+R \Delta_{\mu}+\psi_{S}(R)+\mathcal{L}\{D \circ F\}(R)=\delta I .
$$

Note that

$$
\begin{aligned}
\mathcal{L}\left\{F_{Z}^{(k)}\right\}(s) & =\Delta_{\mu+d^{(k)}}^{-1}\left\{\left(s-R^{(k)}\right)^{-1}\left(e^{-k R^{(k)}}-e^{-s k}\right)\right\} \Delta_{\lambda^{(k)}}, \\
\mathcal{L}\left\{F_{J}^{(k)}+F_{S}^{(k)}\right\}(s) & =\Delta_{\mu+d^{(k)}}^{-1}\left(s-R^{(k)}\right)^{-1}\left[\psi_{S}\left(R^{(k)}\right)-\psi_{S}(s I)+\mathcal{L}\{D \circ F\}\left(R^{(k)}\right)-\mathcal{L}\{D \circ F\}(s I)\right] .
\end{aligned}
$$

Thus,

$$
\begin{aligned}
& \mathcal{L}\left\{g^{(k)}\right\}(s) \\
= & \left(I-\Delta_{\mu+d^{(k)}}^{-1}\left\{\left(s-R^{(k)}\right)^{-1}\left(e^{-k R^{(k)}}-e^{-s k}\right)\right\} \Delta_{\lambda^{(k)}}\right)^{-1} \Delta_{\mu+d^{(k)}}^{-1} \mathcal{L}\left\{\mathcal{T}_{R^{(k)}} \Delta_{\nu}+\mathcal{T}_{R^{(k)}}(D \circ F)\right\} \\
= & \left(\Delta_{\mu+d^{(k)}}-\left\{\left(s-R^{(k)}\right)^{-1}\left(e^{-k R^{(k)}}-e^{-s k}\right)\right\} \Delta_{\lambda^{(k)}}\right)^{-1} \mathcal{L}\left\{\mathcal{T}_{R^{(k)}} \Delta_{\nu}+\mathcal{T}_{R^{(k)}}(D \circ F)\right\} .
\end{aligned}
$$

We note that as $k \rightarrow 0$,

$$
\begin{aligned}
& \Delta_{\mu+d^{(k)}}-\left\{\left(s-R^{(k)}\right)^{-1}\left(e^{-k R^{(k)}}-e^{-s k}\right)\right\} \Delta_{\lambda^{(k)}} \\
= & \Delta_{\mu}+\frac{1}{k} \Delta_{\sigma^{2}}-\left(s-R^{(k)}\right)^{-1}\left[k\left(s-R^{(k)}\right)-\frac{1}{2} k^{2}\left(s^{2}-\left(R^{(k)}\right)^{2}\right)+O\left(k^{3}\right)\right] \Delta_{\lambda^{(k)}} \\
\rightarrow & \left(\Delta_{2 \mu / \sigma^{2}}+R+s\right) \Delta_{\sigma^{2} / 2}=(P+s) \Delta_{\sigma^{2} / 2} .
\end{aligned}
$$

Therefore, as $k \rightarrow 0$,

$$
\mathcal{L} g^{(k)} \rightarrow \mathcal{L}\left\{\Delta_{\sigma^{2} / 2}^{-1} \mathcal{E}_{P}\left\{\mathcal{T}_{R} \Delta_{\nu}+\mathcal{T}_{R}(D \circ F)\right\}\right\}
$$

which implies $g^{(k)}$ converges to $g$ according to Feller [18], Theorem XIII.1.2. This completes the proof of (D.1). 


\section{References}

[1] Hansjörg Albrecher, Corina Constantinescu, Zbigniew Palmowski, Georg Regensburger, and Markus Rosenkranz. Exact and asymptotic results for insurance risk models with surplus-dependent premiums. SIAM J. Appl. Math., 73(1):47-66, 2013.

[2] Hansjörg Albrecher, Corina Constantinescu, Gottlieb Pirsic, Georg Regensburger, and Markus Rosenkranz. An algebraic operator approach to the analysis of Gerber-Shiu functions. Insurance Math. Econom., 46(1):42-51, 2010.

[3] Hansjörg Albrecher and Jevgenijs Ivanovs. A risk model with an observer in a markov environment. Risks, 1(3):148-161, 2013.

[4] Søren Asmussen and Hansjörg Albrecher. Ruin probabilities. Advanced Series on Statistical Science \& Applied Probability, 14. World Scientific Publishing Co. Pte. Ltd., Hackensack, NJ, second edition, 2010.

[5] Florin Avram, Zbigniew Palmowski, and Martijn R. Pistorius. On the optimal dividend problem for a spectrally negative Lévy process. Ann. Appl. Probab., 17(1):156-180, 2007.

[6] Jean Bertoin. Lévy processes, volume 121 of Cambridge Tracts in Mathematics. Cambridge University Press, Cambridge, 1996.

[7] Enrico Biffis and Andreas E. Kyprianou. A note on scale functions and the time value of ruin for Lévy insurance risk processes. Insurance Math. Econom., 46(1):85-91, 2010.

[8] Enrico Biffis and Manuel Morales. On a generalization of the Gerber-Shiu function to path-dependent penalties. Insurance Math. Econom., 46(1):92-97, 2010.

[9] Lothar Breuer. First passage times for Markov additive processes with positive jumps of phase type. J. Appl. Probab., 45(3):779-799, 2008.

[10] Lothar Breuer. Exit problems for reflected Markov-modulated Brownian motion. J. Appl. Probab., 49(3):697-709, 2012.

[11] Jun Cai, Runhuan Feng, and Gordon E. Willmot. On the expectation of total discounted operating costs up to default and its applications. Adv. in Appl. Probab., 41(2):495-522, 2009 .

[12] Erhan Çinlar. Markov renewal theory. Advances in Appl. Probability, 1:123-187, 1969.

[13] Terence Chan, Andreas E. Kyprianou, and Mladen Savov. Smoothness of scale functions for spectrally negative Lévy processes. Probab. Theory Related Fields, 150(3-4):691-708, 2011.

[14] Eric C. K. Cheung and Runhuan Feng. A unified analysis of claim costs up to ruin in a Markovian arrival risk model. Insurance Math. Econom., 53(1):98-109, 2013.

[15] Eric C. K. Cheung and David Landriault. A generalized penalty function with the maximum surplus prior to ruin in a MAP risk model. Insurance Math. Econom., 46(1):127-134, 2010.

[16] Bernardo D'Auria, Jevgenijs Ivanovs, Offer Kella, and Michel Mandjes. First passage of a Markov additive process and generalized Jordan chains. J. Appl. Probab., 47(4):1048-1057, 2010 . 
[17] Eugene B. Dynkin. Markov processes. Vols. I, II, volume 122 of Translated with the authorization and assistance of the author by J. Fabius, V. Greenberg, A. Maitra, G. Majone. Die Grundlehren der Mathematischen Wissenschaften, Bände 121. Academic Press Inc., Publishers, New York, 1965.

[18] William Feller. An introduction to probability theory and its applications. Vol. II. Second edition. John Wiley \& Sons Inc., New York, 1971.

[19] Runhuan Feng. A matrix operator approach to the analysis of ruin-related quantities in the phase-type renewal risk model. Schweiz. Aktuarver. Mitt., (1-2):71-87, 2009.

[20] Runhuan Feng. On the total operating costs up to default in a renewal risk model. Insurance Math. Econom., 45(2):305-314, 2009.

[21] Runhuan Feng. An operator-based approach to the analysis of ruin-related quantities in jump diffusion risk models. Insurance Math. Econom., 48(2):304-313, 2011.

[22] Runhuan Feng and Yasutaka Shimizu. On a generalization from ruin to default in a Lévy insurance risk model. Methodol. Comput. Appl. Probab., 15(4):773-802, 2013.

[23] Hans U. Gerber and Bruno Landry. On the discounted penalty at ruin in a jump-diffusion and the perpetual put option. Insurance Math. Econom., 22(3):263-276, 1998.

[24] Hans U. Gerber and Elias S. W. Shiu. The time value of ruin in a Sparre Andersen model. N. Am. Actuar. J., 9(2):49-84, 2005. With discussion and a reply by the authors.

[25] Chun-Hua Guo. On a quadratic matrix equation associated with an $M$-matrix. IMA $J$. Numer. Anal., 23(1):11-27, 2003.

[26] Roger A. Horn and Charles R. Johnson. Topics in matrix analysis. Cambridge University Press, Cambridge, 1994. Corrected reprint of the 1991 original.

[27] Jevgenijs Ivanovs. Potential measures of one-sided markov additive processes with reflecting and terminating barriers. 2013.

[28] Jevgenijs Ivanovs, Onno Boxma, and Michel Mandjes. Singularities of the matrix exponent of a Markov additive process with one-sided jumps. Stochastic Process. Appl., 120(9):1776$1794,2010$.

[29] Jevgenijs Ivanovs and Zbigniew Palmowski. Occupation densities in solving exit problems for Markov additive processes and their reflections. Stochastic Process. Appl., 122(9):3342$3360,2012$.

[30] Martin Jacobsen. The time to ruin for a class of Markov additive risk process with two-sided jumps. Adv. in Appl. Probab., 37(4):963-992, 2005.

[31] Jean Jacod and Albert N. Shiryaev. Limit theorems for stochastic processes, volume 288 of Grundlehren der Mathematischen Wissenschaften [Fundamental Principles of Mathematical Sciences]. Springer-Verlag, Berlin, second edition, 2003.

[32] Andreas E. Kyprianou. Introductory lectures on fluctuations of Lévy processes with applications. Universitext. Springer-Verlag, Berlin, 2006.

[33] Andreas E. Kyprianou and Zbigniew Palmowski. A martingale review of some fluctuation theory for spectrally negative Lévy processes. In Séminaire de Probabilités XXXVIII, volume 1857 of Lecture Notes in Math., pages 16-29. Springer, Berlin, 2005. 
[34] Andreas E. Kyprianou and Zbigniew Palmowski. Fluctuations of spectrally negative Markov additive processes. In Séminaire de probabilités XLI, volume 1934 of Lecture Notes in Math., pages 121-135. Springer, Berlin, 2008.

[35] Andreas E. Kyprianou and Xiaowen Zhou. General tax structures and the Lévy insurance risk model. J. Appl. Probab., 46(4):1146-1156, 2009.

[36] David Landriault and Gordon Willmot. On the Gerber-Shiu discounted penalty function in the Sparre Andersen model with an arbitrary interclaim time distribution. Insurance Math. Econom., 42(2):600-608, 2008.

[37] Shuanming Li and José Garrido. On a general class of renewal risk process: analysis of the Gerber-Shiu function. Adv. in Appl. Probab., 37(3):836-856, 2005.

[38] Markus Rosenkranz and Georg Regensburger. Solving and factoring boundary problems for linear ordinary differential equations in differential algebras. J. Symbolic Comput., 43(8):515-544, 2008.

[39] Zhimin Zhang and Eric C. K. Cheung. The Markov additive risk process under an Erlangized dividend barrier strategy. Methodol. Comput. Appl. Probab., to appear, 2014. 